\title{
Diseño de un reflector parabólico perfilado para transmisión satelital sobre el territorio mexicano
}

\author{
S. Landeros-Ayala, R. Sámano-Robles y R. Neri-Vela \\ Departamento de Telecomunicaciones \\ División de Ingeniería Eléctrica, Facultad de Ingeniería, UNAM \\ E-mails: sland@dctrl.fi-b.unam.mx,ramiro_samano@yahoo.com, aldeca@ aries.fi-b.unam.mx
}

(recibido: enero de 2003; aceptado: junio de 2003)

\begin{abstract}
Resumen
Los satélites de comunicaciones de este nuevo siglo tienden a emplear, cada vez más, platos parabólicos perfilados e iluminados con una sola corneta para generar huellas de radiación irregulares que se adapten a la geometría de su zona de servicio. Esta novedosa técnica implica la síntesis de deformaciones sobre la superficie del plato, calculadas a través de un proceso iterativo descrito en este artículo. Además de las bases matemáticas para diseñar platos perfilados en cualquier frecuencia y con huellas de cualquier geometría, también se proponen las características de una antena que transmita sobre el territorio mexicano.
\end{abstract}

Descriptores: reflector parabólico perfilado, huella irreg ular, haz conformado, Transformada de Fourier, antena offset.

\begin{abstract}
M oderntelecommunicationssatel lites useshaped par a bolic reflectors to trans mit withir reg ut lar foot prints that cover thegeo graph ical geom etry of their ser vicear eas. This recenttech nique con sistsin syn thesizing small sur faceir regu lari itieson a para bolicdish ill u mi natel by a singlefeed, that arecal cu lated through an it er a tivepro cess described in this pa per. All the mathemat i cal procedure, for any fre quency or foot print shape, is given here, as well as the char at ter is tics of a shaped dish pro posed for cov er age over M ex ico.
\end{abstract}

Keywords: shaped par a bolicreflector, ir reg u lar foot print, con toured beam, Fou rier Transform, 0 ffset antenna.

\section{Introducción}

Los reflectores parabólicos perfilados, iluminados con una sola corneta, son utilizados actualmente en satélites de comunicaciones para obtener huellas conformadas de radiación. Su uso ha suplantado progresivamente a los alimentadores hechos con arreglos de decenas de cornetas, que implicaban antenas muy costosas y voluminosas.

Muchas de las antenas de los satélites geoestacionarios no tienen una radiación simétrica alrededor de su eje, sino que el patrón de radiación de sus ganancias en 
dirección hacia la superficie de la Tierra está conformado de tal manera que cubra eficientemente su zona de servicio. Para dichas antenas, su directividad se representa en los mapas de la zona de servicio como proyecciones de contornos de igual ganancia (Neri, 2003). Las antenas utilizadas para este tipo de cobertura se denominan de haz conformado. En la figura 1 se muestra la huella de radiación de un satélite europeo.

Las antenas reflectoras son ideales para obtener haces con huellas de iluminación conformadas. Generalmente, se utiliza la configuración de reflector con alimentación descentrada (offset, en inglés). Este arreglo elimina la desventaja del bloqueo por alimentador y está constituido por una sección descentrada de un paraboloide cuyo foco queda fuera de la trayectoria de las ondas reflejadas. En la figura 2, se muestra un reflector parabólico visto de frente y la zona sombreada corresponde a lo que sería una antena tipo offset, que no emplea todo el plato, sino sólo una porción de éste. Para sostener el alimentador en la antena offset, se utiliza un brazo que sale por debajo del reflector (Figura 3), de manera que ni la unidad exterior ni el brazo que la sustenta proyectan sombra alguna sobre el plato, es decir, ninguna interferencia por bloqueo. El rendimiento de las antenas offset alcanza el $65 \%$, con lo cual, para igual ganancia, el diámetro del reflector es menor que el de las antenas de foco primario, mismas que sí sufren de bloqueo.

Existen varias formas para crear patrones de radiación asimétricos. La manera convencional del siglo XX usaba arreglos de antenas de corneta, cada una de ellas alimentada con una amplitud y fase de señal determinada, que al sumarse vectorialmente modificaban el patrón de radiación para formar la huella deseada (Figura 4a). La última y más reciente técnica para obtener patrones de radiación asimétricos emplea un reflector parabólico perfilado con una sola corneta como alimentador (Figura 4b).

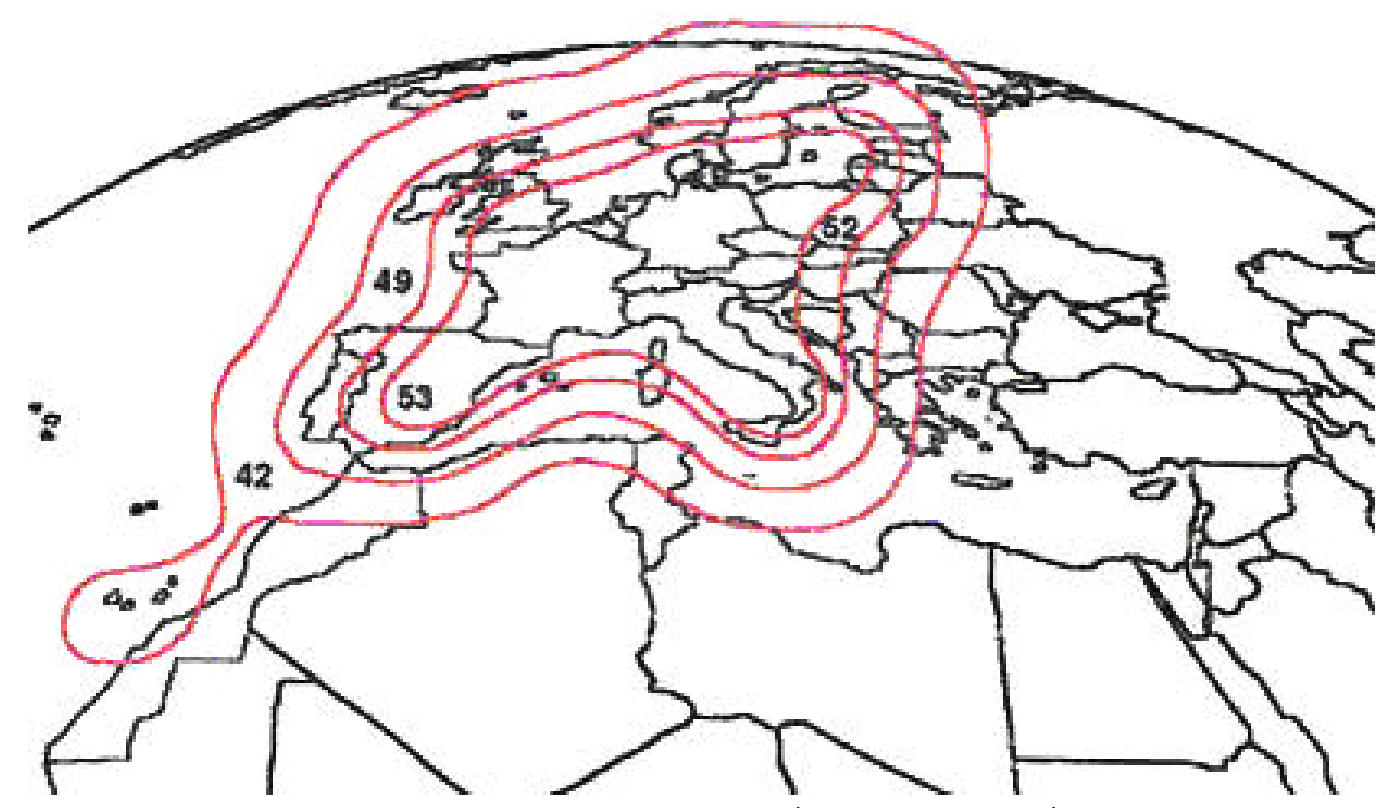

Figura 1. Huella de radiación para Europa en la banda Ka (Satélite H otbird). Los valores indicados de PIRE (Potencia Isotrópica Radiada Efectiva) para cada contorno están en dBW e incluyen la ganancia de la antena y la potencia del transpondedor en dirección hacia los puntos de dicho contorno 


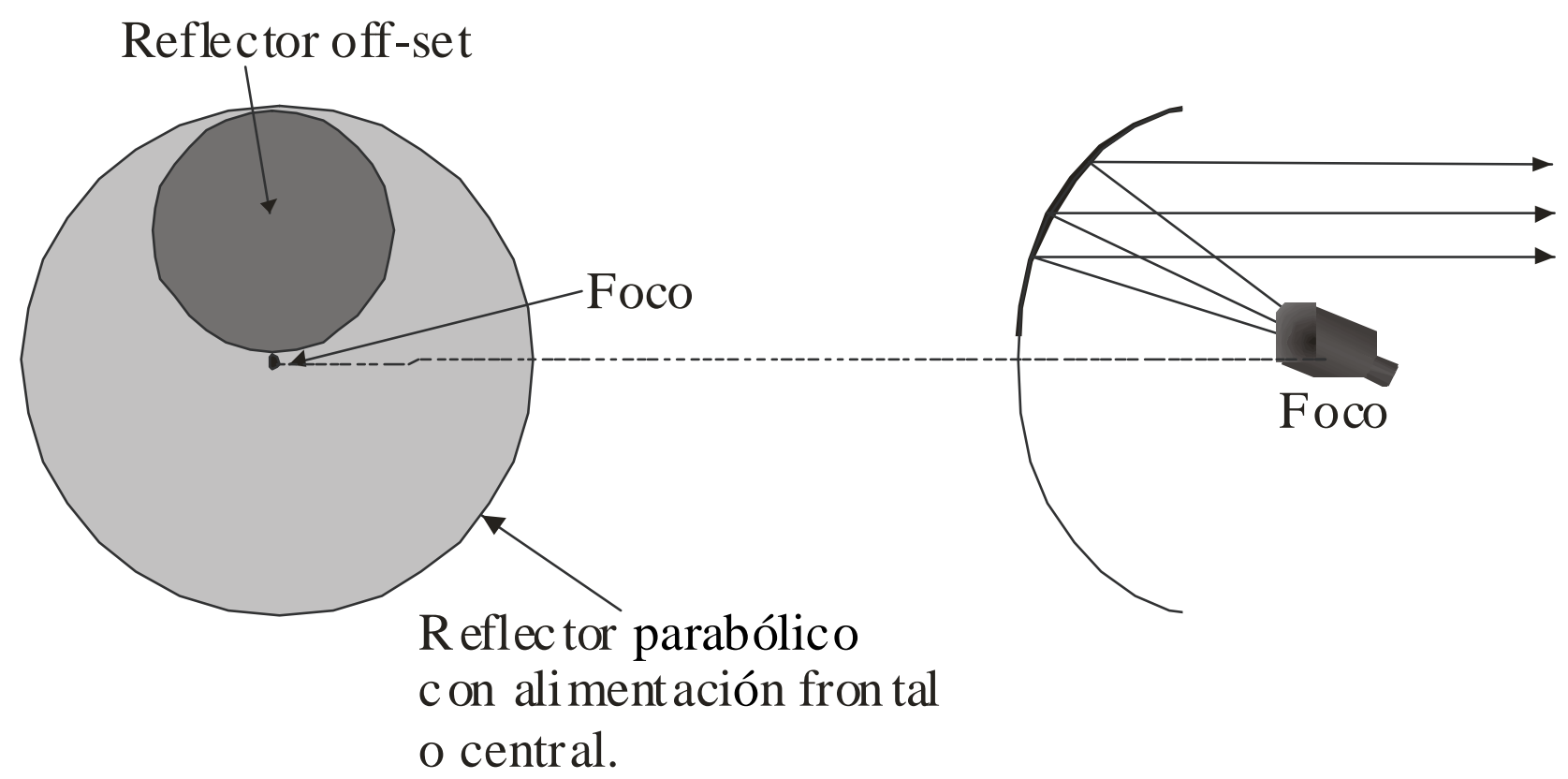

Figura 2. Vista frontal y lateral de un reflector parabólico tipo offset y su relación con un reflector parabólico de alimentación central

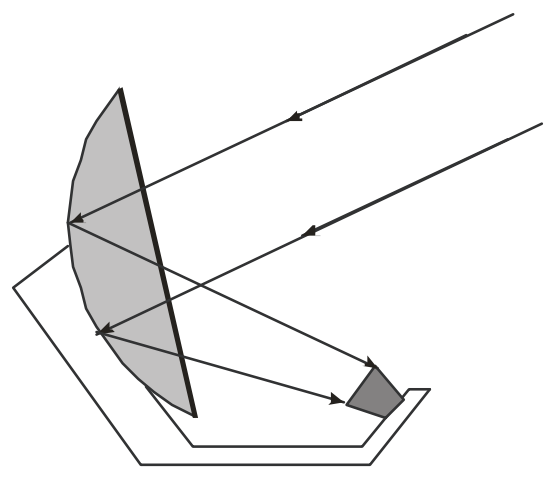

Figura 3. Zonas de iluminación y radiación de un reflector offset 


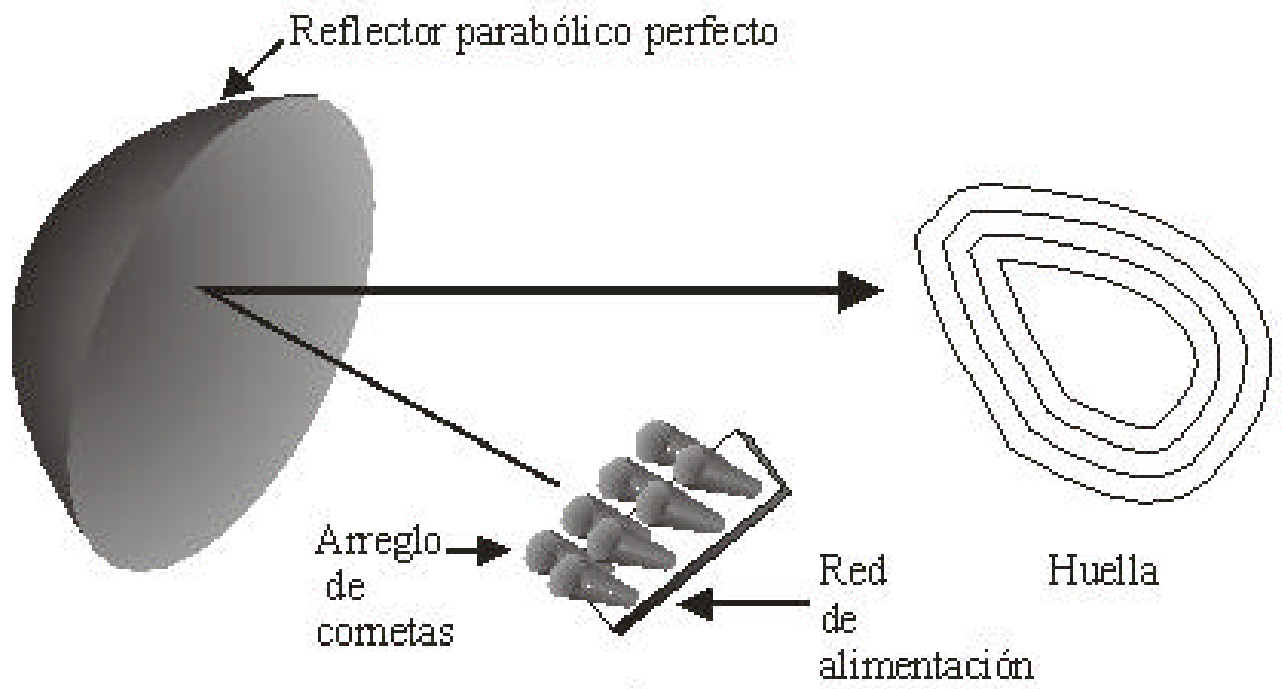

a)

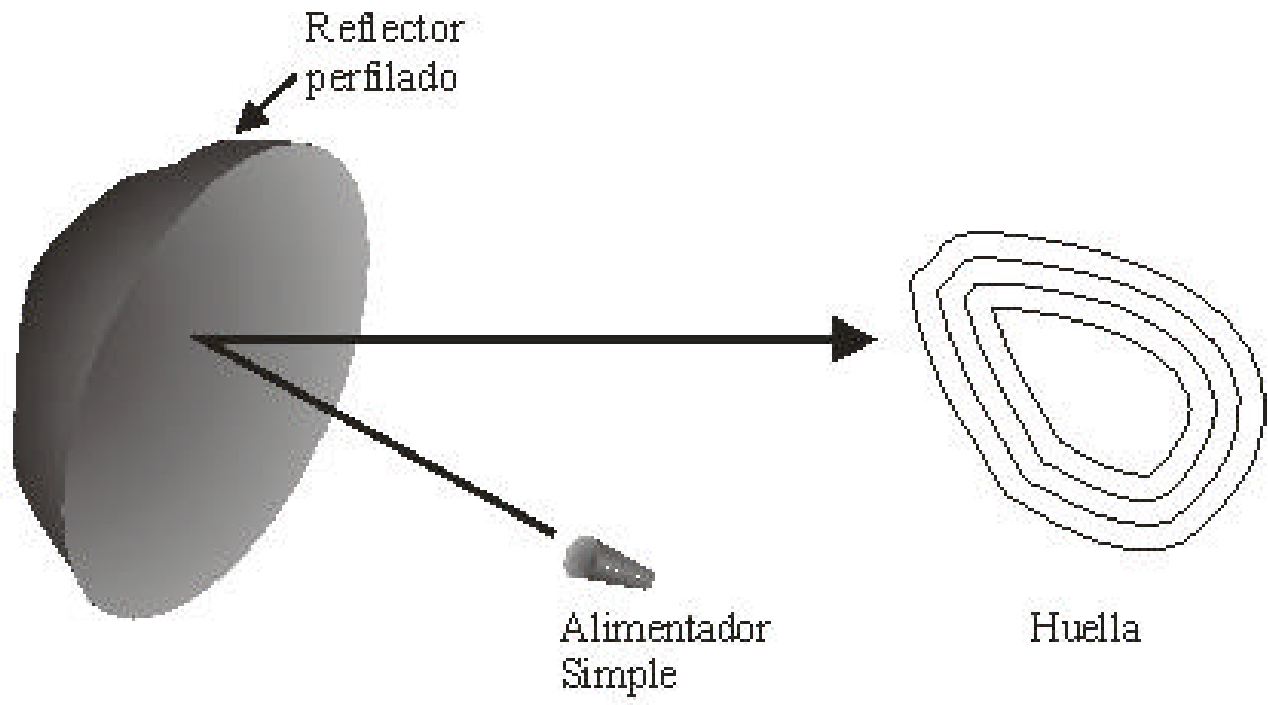

b)

Figura 4. Técnicas para producir huellas de radiación conformadas: a) A rreglo de antenas de corneta con amplitud y fase variables y un reflector parabólico perfecto; b) Reflector parabólico perfilado con una sola corneta como alimentador 
La técnica del reflector parabólico perfilado con un solo alimentador ha sido descrita por varios autores (Cherrette y Acosta, 1989), (Shogen et al., 1992), (Shoki y Kawabata 1993), (Pinsard et al., 1997) y a partir de los años noventas es la más utilizada en los satélites de comunicaciones. Además de formar huellas de radiación muy aproximadas a las características geográficas de las zonas de cobertura, disminuye el costo comparado con el de los arreglos de fase que son muy voluminosos, por lo que las redes de alimentación que eran necesarias para tales arreglos se eliminan. El método consiste en modificar la superficie del reflector, con crestas y hendiduras en diferentes puntos para alterar la fase y dirección de los rayos reflejados por el mismo, obteniéndose así el mismo efecto que con la técnica del pasado.

El procedimiento de diseño de un reflector parabólico perfilado con alimentador simple consiste básicamente en los siguientes pasos:

1) Se definen la región de cobertura y las direcciones de optimización, así como los niveles de ganancia deseados para diferentes puntos. Al mismo tiempo, se aproximan las dimensiones del reflector de acuerdo al ancho del haz de media potencia.

2) Se plantean un modelo inicial del reflector parabólico perfecto y las características de su alimentador (distribución de amplitud y fase iniciales, así como la definición de las dimensiones iniciales del reflector).

3) Se optimiza la distribución de fase, minimizando una función de costo ${ }^{1}$.
4) Se calcula la nueva superficie del reflector perfilado de acuerdo a la distribución de fase optimizada en el paso anterior.

5) Se obtiene la nueva distribución de amplitud en el plano de análisis de acuerdo a la superficie calculada del reflector en el paso 4.

6) Se repite iterativamente el proceso desde el punto 3 al punto 5, hasta que el patrón obtenido se aproxime lo mejor posible al deseado.

A continuación, se de scribe la secuencia del análisis matemático necesario para poder sintetizar tal antena, iniciando con la selección del área de cobertura.

\section{Análisis matemático (1 a Parte)}

El primer paso consiste en definir la región de cobertura deseada, por ejemplo un país, isla 0 continente, los cuales por lo general tienen una forma irregular. Como aproximación inicial, tomando en cuenta la figura 5, se puede saber el diámetro requerido del reflector, considerando que la ganancia en el borde 0 contorno del área de cobertura sea de $-3 \mathrm{~dB}$ por debajo de la máxima ganancia del reflector. Con la ecuación (1), se puede relacionar el ancho del haz de media potencia con el diámetro de un re flec tor (Neri, 2003):

$$
B W_{-3 d B} \approx 75\left(\frac{\lambda}{D}\right) \text { grados }
$$

1 Una función de costo es cualquier función de una o más vari ables, cuyo valor se busca minimizar lo más posible. En este caso partic ular, la función de costo se forma de una diferencia de cuadrados de la ganancia obtenida menos la ganancia deseada hacia las direcciones de optimización. 
Donde $\lambda$ es la longitud de onda y $D$ es el diámetro del reflec tor.

Respecto al área de cobertura, debe tomarse en cuenta que los puntos definidos para el contorno de dicha área no pueden tener formas muy complicadas, debido a que el reflector, y en especial, la distribución de fase, se verían afectadas al tener que cumplir con condiciones muy rigurosas y discontinuidades muy pronunciadas. Es conveniente, por lo tanto, definir al contorno con una forma sim ple y suave, sin cambios bruscos y con un número limitado de puntos, sólo los necesarios para definir la figura básica. Este comentario es importante, ya que se omite en la literatura disponible en inglés y si no se toma en cuenta, puede conducir a esfuerzos inútiles y mucho consumo de tiempo de cómputo por investigadores que se estén involucrando por primera vez con esta técnica. También es importante recalcar que la ganancia deseada sobre los puntos de optimización tiene un límite inferior y uno su pe rior (no hay una relación analítica) para el cual el método converge de manera satisfactoria. Fuera de este rango, el método resulta inestable. Por ello, resulta necesario que el nivel de ganancia deseada se encuentre cercano a un promedio aproximado de la ganancia inicial del re flec tor ideal sobre dichos puntos.

Continuando con el desarrollo matemático, se plantean las fórmulas para representar las direcciones espaciales y el área de cobertura vista desde el satélite. Para esto, resulta conveniente colocar un marco de referencia global, como se muestra en la figura $6 a$. Se considera que el satélite está ubicado a la misma longitud que el punto central de la huella donde se quiere obtener la radiación. En la práctica, la zona geográfica de servicio tiene una longitud más hacia el este que la del satélite, pero esto se corrige orientando la antena. No es el mismo caso para las lat i tudes, pero como aproximación inicial conviene

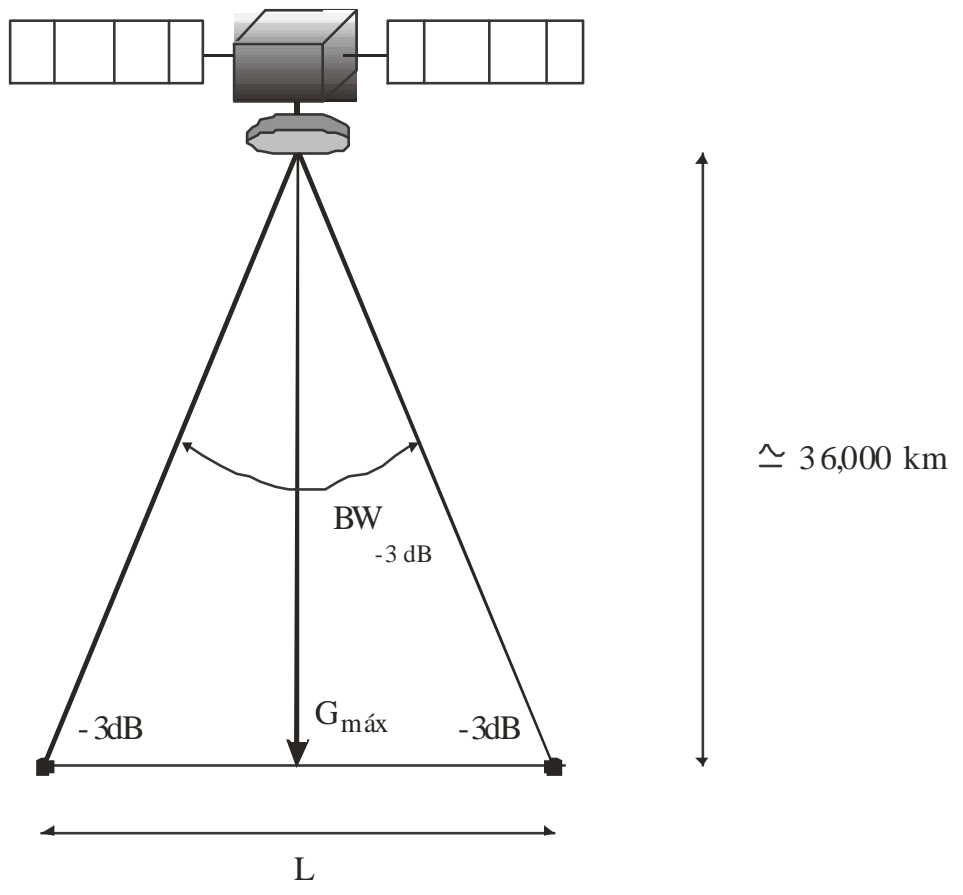

Figura 5. D efinición de la zona de cobertura. L es cercana a la dimensión más larga de la zona 
considerar que la antena del satélite apunta directamente hacia la línea ecuatorial. Una vez definidas las coordenadas de los puntos de análisis y del punto central C (véase más adelante la Fig. 17), que inicialmente recibe el máximo de radiación, se realiza una rotación del sistema de referencia para apuntar hacia la latitud deseada (Figura 6b).

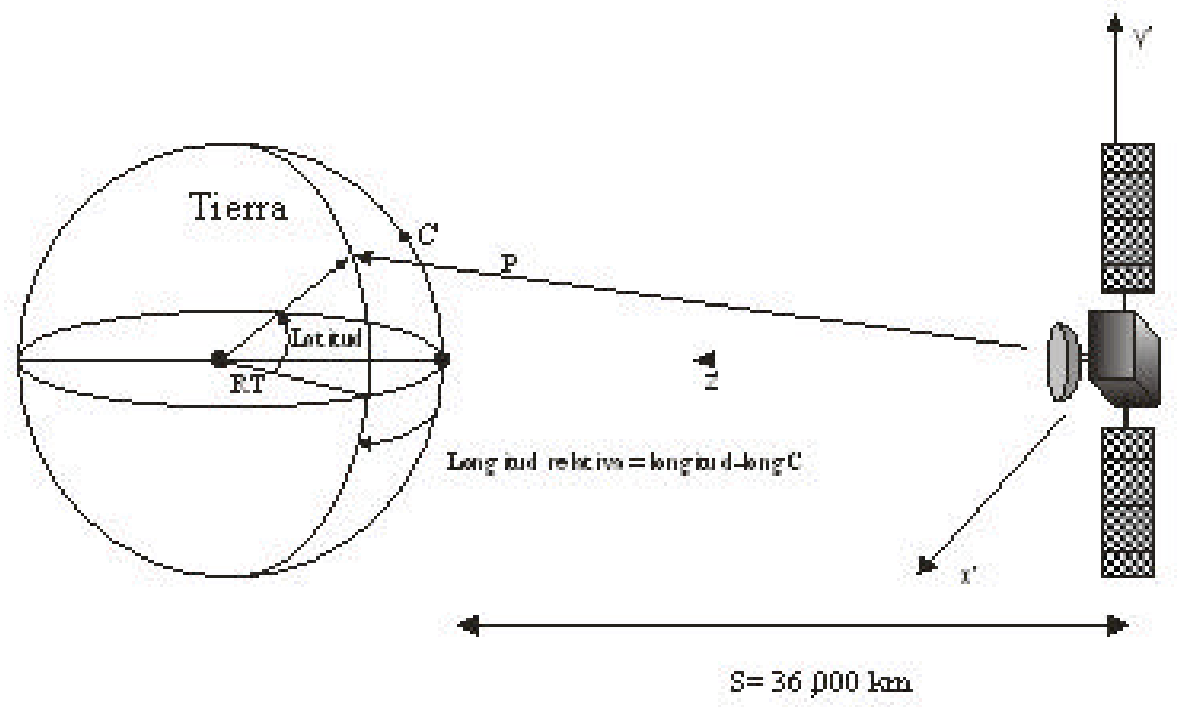

(a)

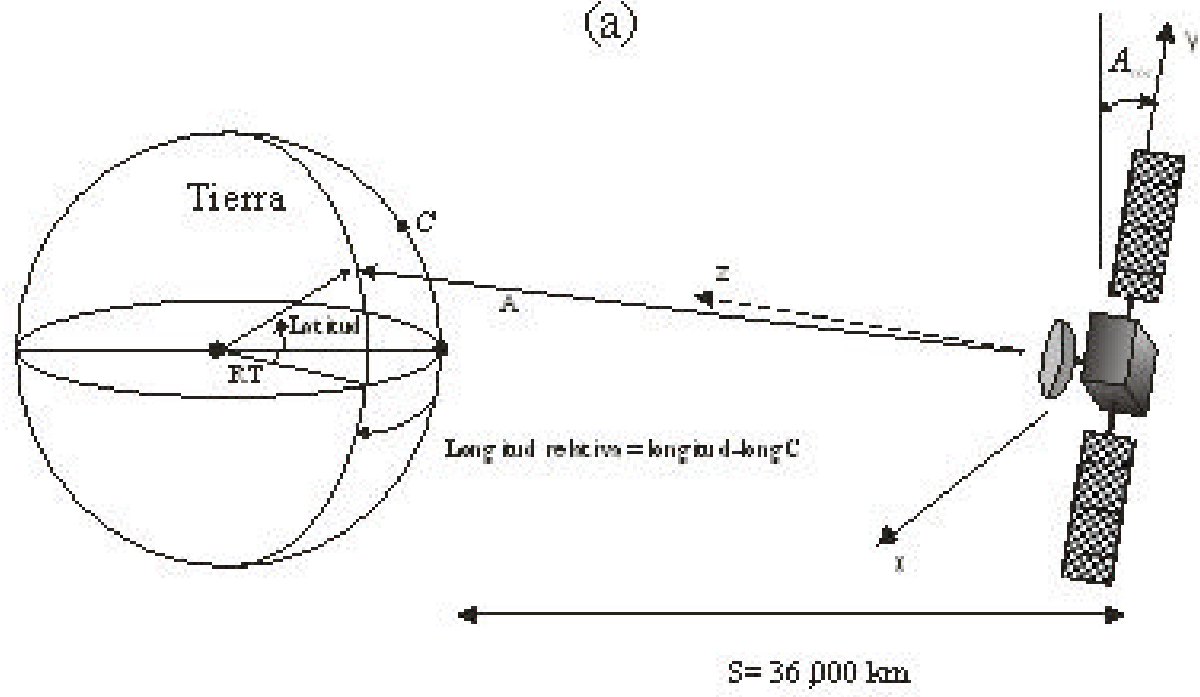

(b)

Figura 6. (a) Marco de referencia global para definir direcciones desde un satélite geoestacionario hacia un punto terrestre. El vector de posición P define la ubicación de un punto del área de cobertura y su latitud geográfica. (b) M arco de referencia del satélite rotado por un ángulo $\mathrm{A}_{\text {rot }}$ sobre el plano yz para que el eje del plato apunte hacia el punto 
Considerando inicialmente que el satélite apunta hacia el ecuador y que el punto de máxima radiación es $C$ (long $C$, lat $C$ ), se pueden definir los demás puntos de análisis sobre la superficie de la Tierra, representados por el vec tor de posición $\mathbf{P}$, cuyas componentes son:

$P_{x}=R T \cos ($ latitud $) \operatorname{sen}($ longitud $-\operatorname{long} C)$

(2)

$P_{y}=R T \operatorname{sen}($ latitud $)$

$P_{z}=(S+R T)-R T \cos ($ latitud $) \cos ($ long itud - long $C)$

Donde:

$R T=$ Radio de la Tierra

$S=$ Distancia del satélite a la superficie de la Tierra.

La latitud y longitud son las coordenadas de cualquier punto de análisis sobre la superficie de la Tierra. La magnitud del vector $\mathbf{P}$ está dada por:

$$
\mathbf{P}=\sqrt{P_{x}^{2}+P_{y}^{2}+P_{z}^{2}}
$$

Y sus componentes normalizadas son:

(6)

$$
R_{x}=\frac{P_{x}}{\mathbf{P}}
$$

$$
R_{y}=\frac{P_{y}}{\mathbf{P}}
$$

$$
R_{z}=\frac{P_{z}}{\mid \mathbf{P}}
$$

Cuando el satélite apunta hacia una latitud diferente que la del ec ua dor, el sistema se rota y la conversión para las nuevas coordenadas $A_{x} A_{y}$ y $A_{z}$ queda de la siguiente forma:

$$
\begin{gathered}
A_{r o t}=\arctan \left(\frac{R T \operatorname{sen}(\text { lat } C)}{S+R T(1-\cos (\text { latC }))}\right) \\
A_{x}=R_{x^{\prime}} \\
A_{y}=R_{y^{\prime}} \operatorname{sen}\left(A_{r o t}\right)+R_{z^{\prime}} \cos \left(A_{r a}\right) \\
A_{z}=R_{y^{\prime}} \cos \left(A_{r o t}\right)-R_{z^{\prime}} \operatorname{sen}\left(A_{r \alpha}\right)
\end{gathered}
$$

De las ecuaciones anteriores pueden obtenerse las direcciones teta y fi de un sistema de coordenadas esféricas:

$$
\text { teta }=\arccos \left(A_{2}\right)
$$

$$
f i=\arctan \left(\frac{A_{y}}{A_{x}}\right)
$$

Los valores anteriores para teta y fi se obtienen para cada punto de análisis sobre la superficie de la Tierra. Si se desea convertir a valores de azimut y elevación (véase más adelante la Fig.15) se procede de la siguiente forma:

$$
\begin{gathered}
\text { Azimut }=-\arctan \left(\frac{A_{x}}{A_{z}}\right) \\
\text { Elevación }=\arctan \left(\frac{A_{y}}{A_{z}}\right)
\end{gathered}
$$

Una vez definidas las direcciones deseadas de optimización y con los datos del área 
geográfica, se procede a modelar el sistema de alimentador-reflector. El alimentador más comúnmente usado para reflectores parabólicos es la antena de corneta. Existen diferentes tipos de antenas de corneta: de paredes lisas, de paredes corrugadas, piramidales, diagonales, cónicas, sectoriales, de fronteras soft y fronteras hard (Clarricoats,1984). Los tipos de corneta más comunes para aplicaciones de reflectores parabólicos son las antenas cónicas corrugadas, que se caracterizan por tener patrones de radiación de baja polarización cruzada y simetría circular casi perfecta. Para modelar el patrón de radiación del alimentador en el programa del reflector perfilado, se recurre a una aproximación de tipo cosinusoidal (Figura 7):

$$
e\left(\theta_{0}\right)=\left[\cos \left(\theta_{0}\right)\right]^{q}
$$

En la figura 8 se muestran cortes del patrón de radiación para algunos exponentes q de la ecuación (17), en comparación con el patrón de una apertura circular alimentada con el modo fundamental de una guía de ondas circular corrugada $\mathrm{HE}_{11}$. Las características del alimentador, como son su ancho del haz de media potencia y los niveles de sus lóbulos laterales dependen de la configuración del reflector a utilizar, de su relación f/D (distancia focal/diámetro) y de la eficiencia de iluminación.

Por lo que se refiere al modelado del re flector perfilado, es necesario definir un marco de referencia apropiado para el análisis de los patrones de radiación y de su proyección sobre la superficie terrestre. Como primera consideración, conviene colocar al foco en el origen del sistema de coordenadas. También por conveniencia, el eje hacia donde se dirige la máxima radiación del reflector parabólico inicial (superficie sin alteraciones) es el eje $z+$, apuntando hacia un plano de análisis localizado en $z=0$ (Figuras 9 a 11). El plano de análisis es el plano donde se evalúan las fases y amplitudes del sistema alimentador- reflector, a partir de las cuales se calculan los patrones de radiación. También puede ser otro plano paralelo al anterior y ubicado a una distancia sobre el eje z mayor (Figura 11).

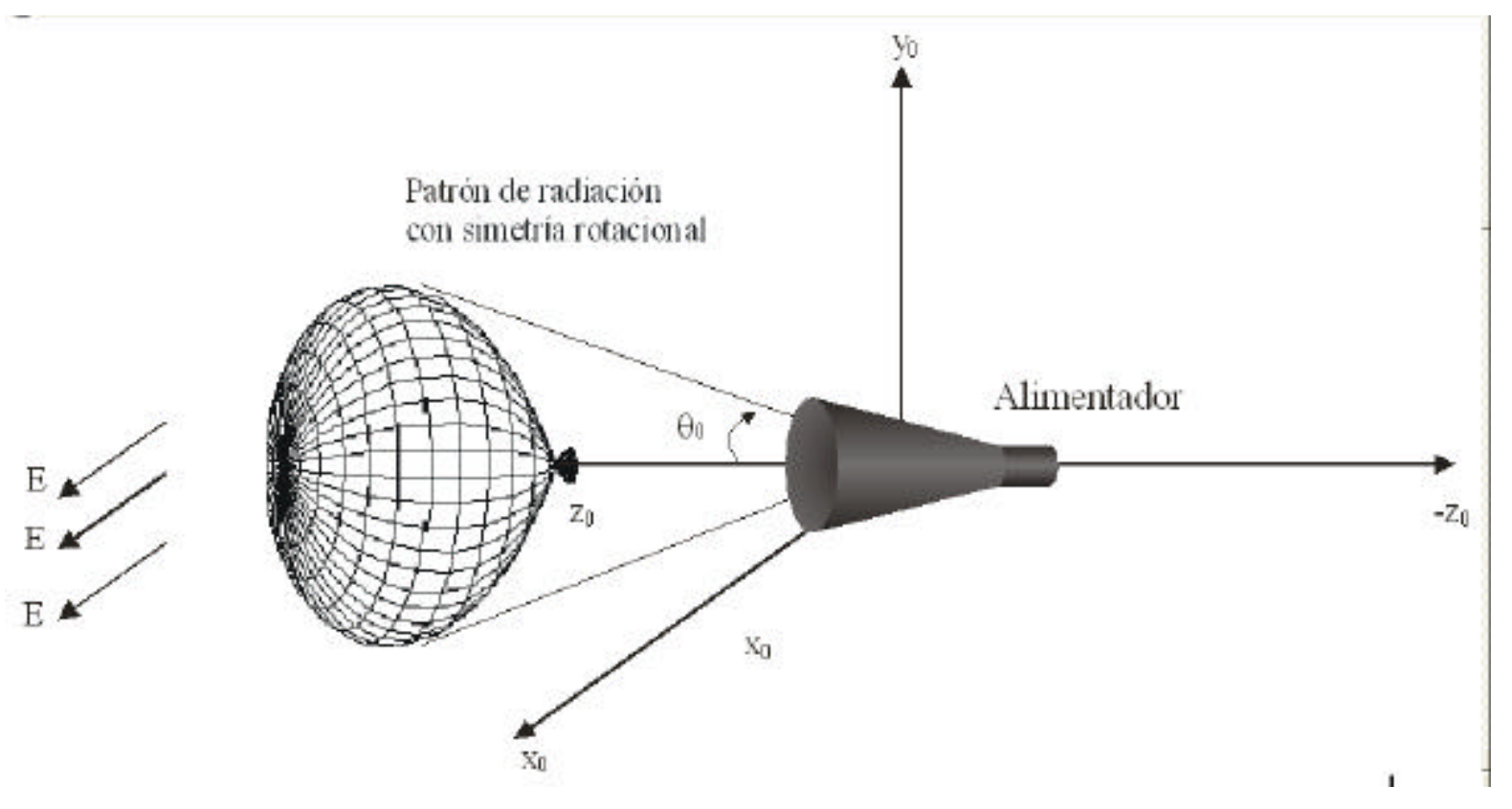

Figura 7. Patrón de radiación del alimentador de un reflector parabólico 


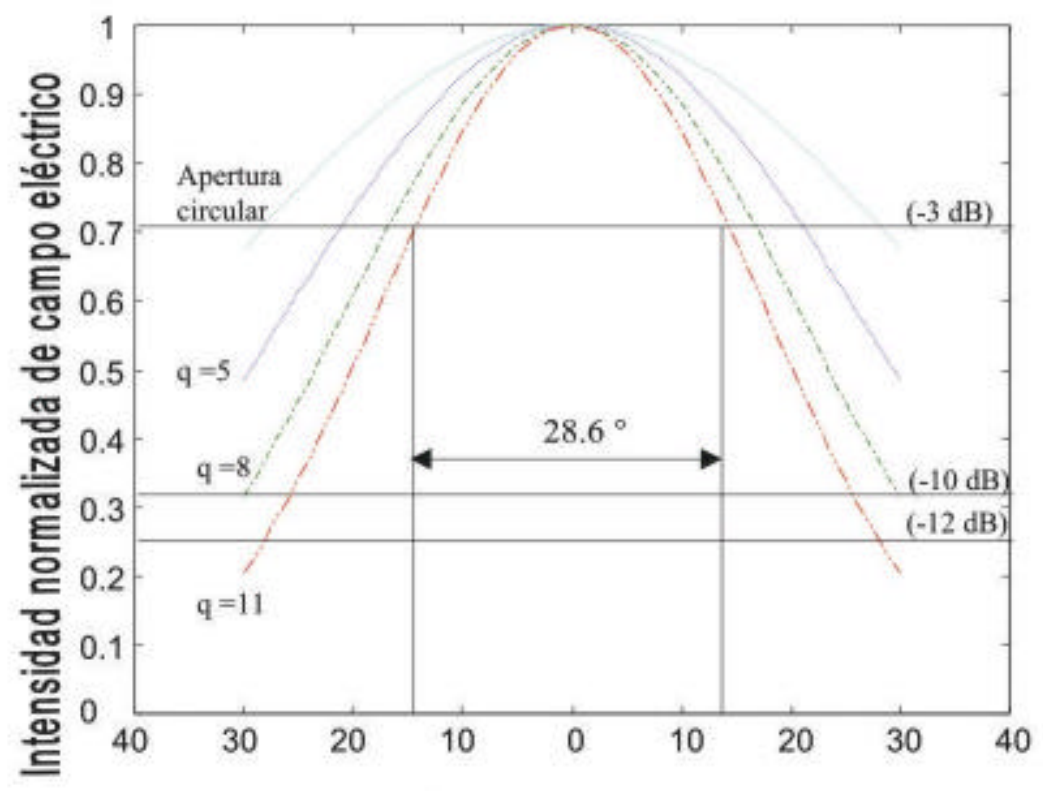

Ángulo $\theta_{\theta}$ [grados]

Figura 8. Comparación de diferentes modelos matemáticos para describir el patrón de radiación del alimentador de un reflector parabólico, con diferente exponente q en la ecuación (17)

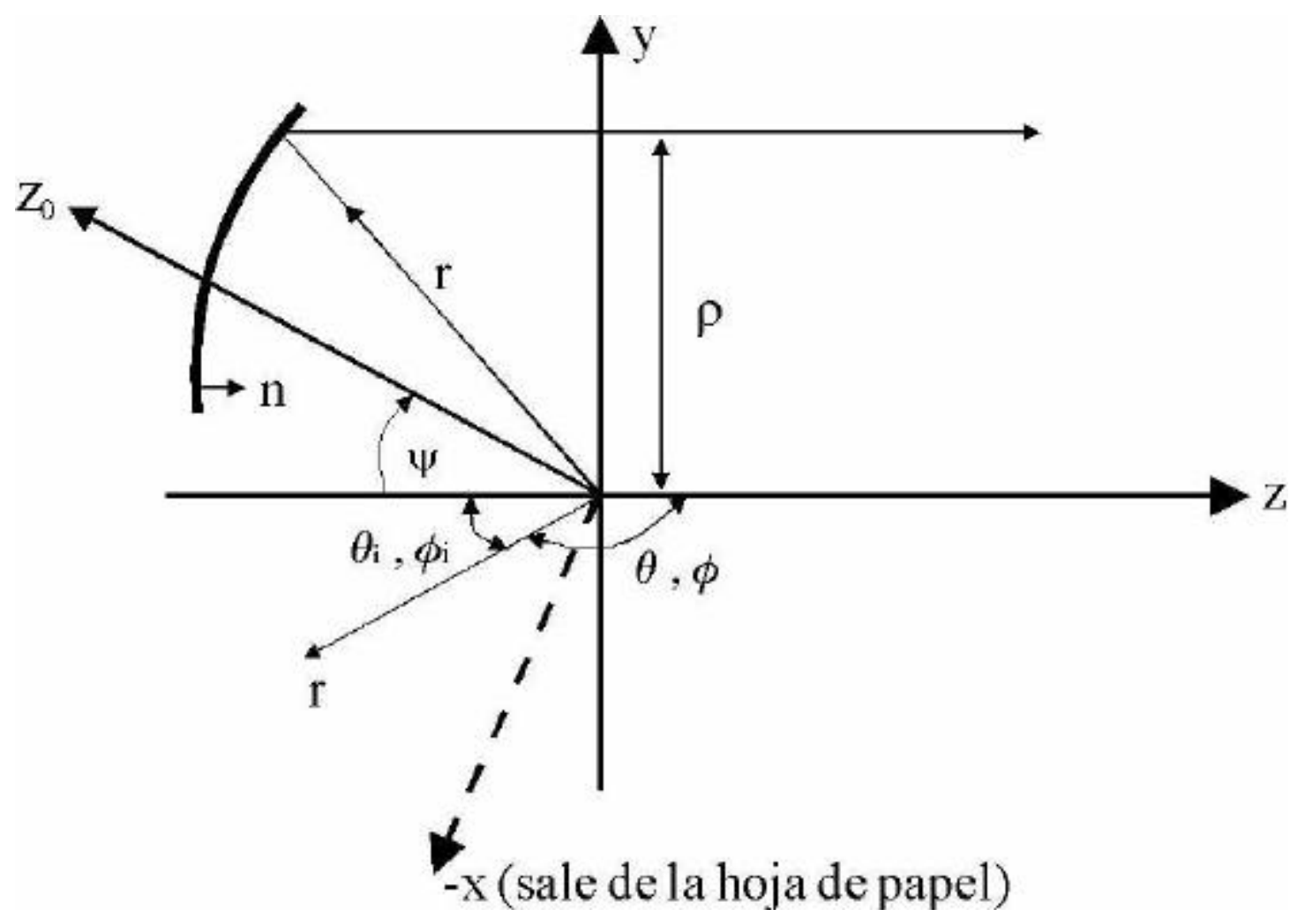

Figura 9. Marco de referencia general para el análisis del reflector parabólico tipo offset 


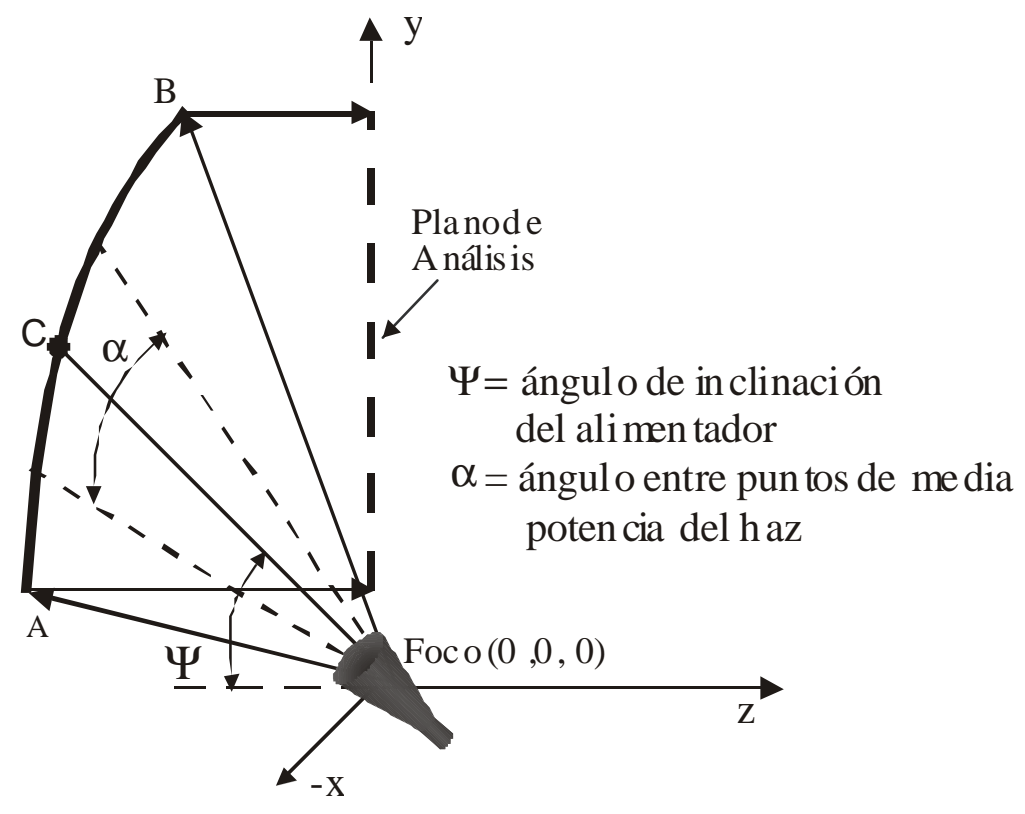

Figura 10. Detalle del modelado geométrico del reflector offset con su alimentador inclinado y el plano de análisis en $z=0$

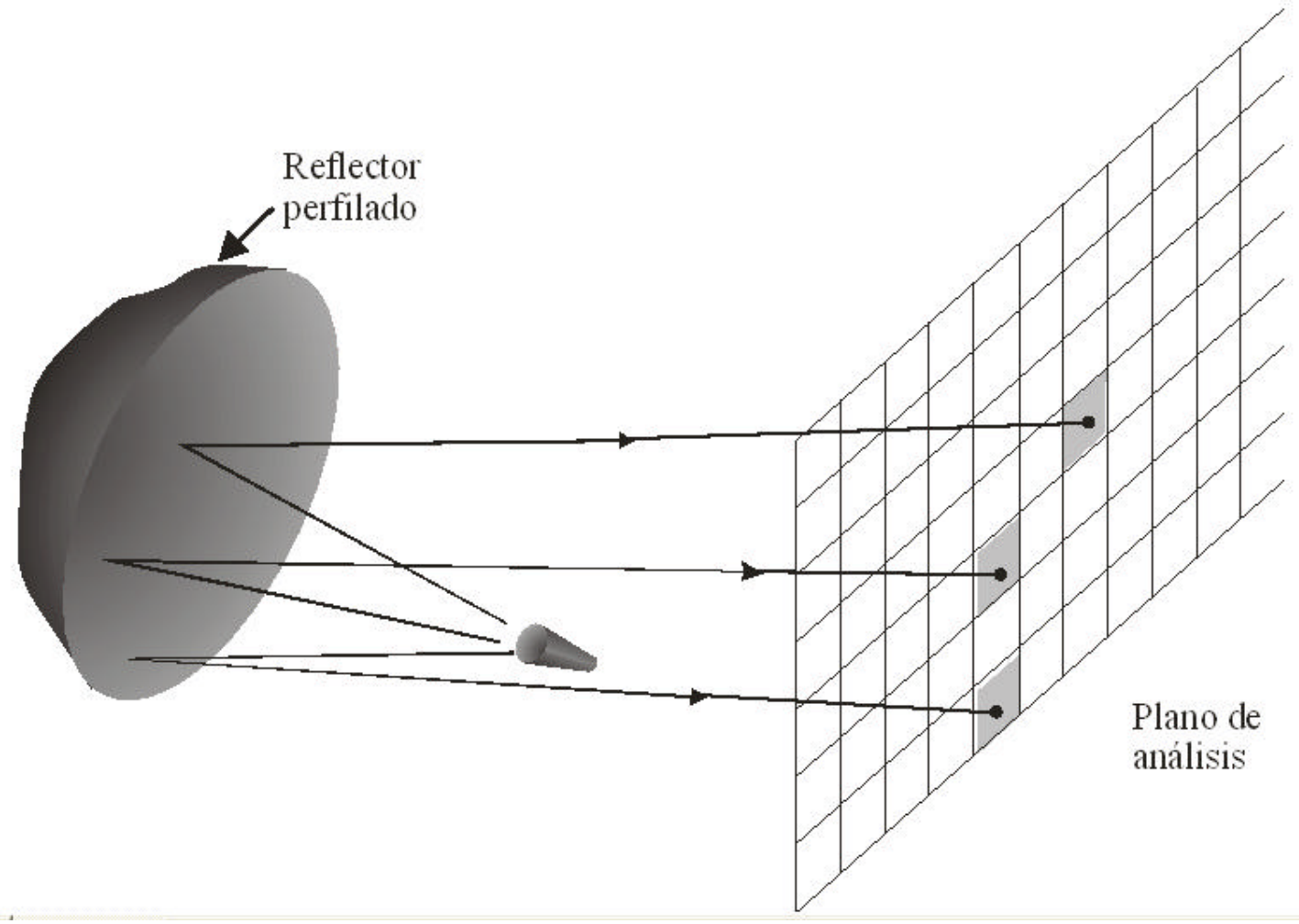

Figura 11. Ilustración de la utilidad del plano de análisis para calcular las ampli tudes y fases radiadas por el sistema alimentador-reflector 
El paraboloide inicial está descrito por la ecuación (18):

$$
z=F(x, y)=\frac{x^{2}+y^{2}}{4 f}-f
$$

0 bien por:

$$
r=\frac{2 f}{1+\cos \theta_{i}}
$$

Donde:

$F=$ valor en el eje $z$ de la superficie del paraboloide que describe la forma del reflector.

$\mathrm{f}=$ distancia focal del plato parabólico.

$\theta_{i}=$ dirección espacial esférica medida desde el eje z-.

$r=$ magnitud del vector de posición $\mathbf{r}$ sobre cualquier punto de la superficie del plato (Figura 9).
El tipo offset se modela al cortar el paraboloide descrito por las ecuaciones 180 19 con el plano dado por la ecuación 20:

$$
y=a(z-b)
$$

Donde a es la pendiente del plano en el eje $y-z$ y b es la intersección de dicho plano con el eje z. Despejando z y sustituyendo en (18), se tiene:

$$
\frac{y}{a}+b=\frac{x^{2}+y^{2}}{4 f}-f
$$

\section{Yordenando (21):}

$$
x^{2}+\left(y-\frac{2 f}{a}\right)^{2}=4 f^{2}\left(1+\frac{1}{a^{2}}\right)+4 f b
$$

Que tiene la forma general $\mathrm{x}^{2}+(\mathrm{y}-H)^{2}=R^{2}$. Esta es la ecuación de una circunferencia proyectada en el plano xy de radio $\mathrm{R}$ (diámetro D) y desplazada por $\mathrm{H}$ en el eje y (Figura 12). El campo radiado por la configuración reflector-alimentador del tipo offset tiene su com-

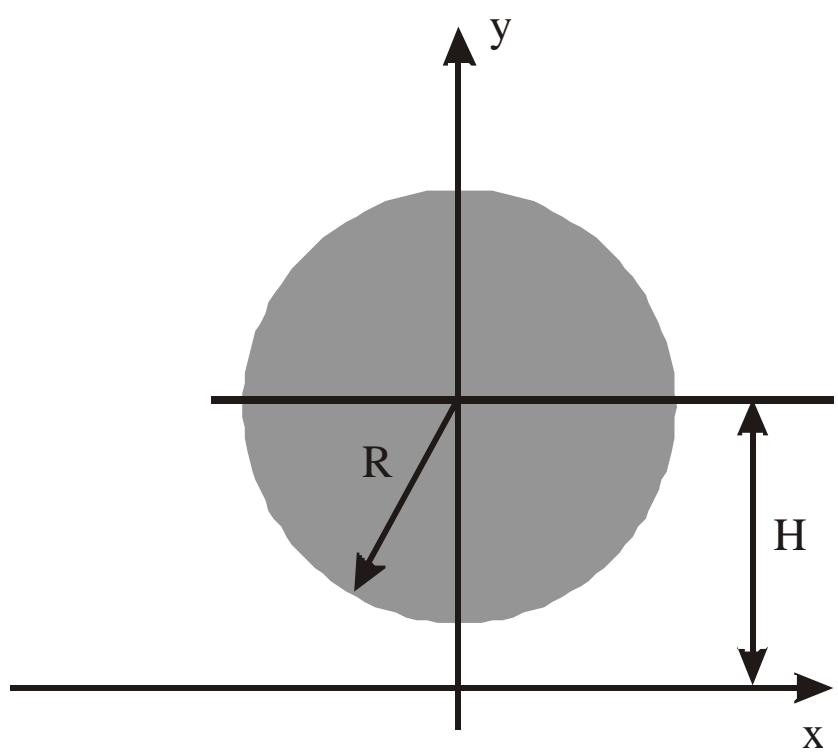

Figura 12. Vista frontal del reflector parabólico offset 
ponente copolarizada (Collin, 1985) expresada de la siguiente forma (Figuras 9 a 11):

$E_{y}=\left[\frac{4 f \cdot e\left(\theta_{0}\right) \cdot e^{-\rho k_{0} f}}{\left(4 f^{2}+\rho^{2}\right)\left[4 f^{2}+\rho^{2}-4 f y \sin \psi+\left(4 f^{2}-\rho^{2}\right) \cos \psi\right]}\right] X$ $\left[\left(4 f^{2}+\rho^{2}-2 x^{2}\right)+\left(4 f^{2}-\rho^{2}+2 x^{2}\right) \cos \psi-4 f y \sin \psi\right]$

Donde

$$
\begin{gathered}
\rho=x^{2}+y^{2} \\
\theta_{0}=a \cos \left(\frac{4 f^{2}-\rho^{2}}{4 f^{2}+\rho^{2}} \cos \psi-\frac{4 f y}{4 f^{2}+\rho^{2}} \sin \psi\right)
\end{gathered}
$$

El ángulo $\theta_{0}$ es igual al ángulo medido a partir del eje cen tral del alimentador, según se muestra en la figura 7. Si el ángulo de inclinación del alimentador es pequeño, el término de polarización cruzada, que resulta en los reflectores tipo offset, puede ser despreciado. Veamos ahora cómo calcular la ganancia del reflector parabólico en una dirección determinada.

\section{Análisis matemático (2 a parte)}

Una vez definidas las condiciones iniciales del re flec tor y las direcciones espaciales sobre las cuales se quiere calcular y optimizar la ganancia del sistema, se procede a calcular la distribución de intensidad de campo y fase producida por el reflector y el alimentador en el plano de análisis. Para ello, conviene utilizar el método de la Transformada de Fourier (Sámano et al., 2002). El plano de análisis es dividido en rejillas cuadradas de dimensiones dxd, donde:

$$
d=\frac{D}{N}
$$

Donde:

$D=$ diámetro del re flec tor parabólico.

$N=$ número de elementos por lado del plano de análisis.

Para modelar la fase, la amplitud y las coordenadas de la superficie del plato calculado, se usan matrices de NxN. De la teoría de antenas de apertura y con la ayuda del método de la Transformada de Fourier, la ganancia de cualquier antena de apertura es (Volakis, 1998):

$$
G_{j}=4 \pi \frac{\left|f_{y}\right|^{2}{ }_{j}}{\int_{s} E_{y}^{2} d x d y}
$$

Donde:

$G_{i}=$ ganancia del re flec tor en la dirección $\mathrm{j}$.

$\mathrm{f}_{\mathrm{y}}=$ Transformada de Fourier de la componente copolarizada y.

$E_{y}=$ componente copolarizada del campo eléctrico sobre el plano de análisis.

$S=$ apertura de la antena.

Considerando la división del plano de análisis en $\mathrm{N} \times \mathrm{N}$ elementos, cada uno como una amplitud y fase constantes, y utilizando el principio de multiplicación de patrones, se tiene:

$$
f_{y_{j}}=\left[\int_{-d / 2-d / 2}^{d / 2} \int_{d / 2}^{d / 2} e^{j k_{x} x+j k_{y} y} d y d x\right]
$$




$$
\sum_{l=1}^{N} \sum_{m=1}^{N} A_{l m} e^{j \beta(l, m)+j k_{x} x(l, m)+j k_{y} y(l, m)}
$$

Donde:

$A_{l m}=$ es la magnitud del campo eléctrico producido por el reflector en el elemento $(I, m)$.

$k_{\mathrm{x}}=\mathrm{k} \operatorname{sen} \theta \cos \phi$.

$k_{y}=k \operatorname{sen} \theta \operatorname{sen} \phi$.

$\beta(l, m)=$ fase del elemento $(I, m)$.

Nótese que el término entre paréntesis, en la ecuación (28), es el patrón de un elemento del arreglo y el término de sumatoria el patrón del arreglo.

$$
\begin{aligned}
& \left.f_{y}\right|_{j}=d^{2} \frac{\operatorname{sen}(u)}{u} \frac{\operatorname{sen}(v)}{v} \\
& \sum_{l=1}^{N} \sum_{m=1}^{N} A_{l m} e^{j \beta(l, m)+j k_{x} x(l, m)+j k_{y} y(l, m)} \\
& G_{j}=4 \pi \frac{16\left(\frac{\operatorname{sen}(u)}{k_{x}} \frac{\operatorname{sen}(v)}{k_{y}}\right)^{2}\left(\sum_{l=1}^{N} \sum_{m=1}^{N} A_{l m} e^{j \beta(l, m)+j k_{x} x(l, m)+k_{y} y(l, m)}\right)^{2}}{\sum_{l=1}^{N} \sum_{m=1}^{N}\left[A_{l m}\right]^{2} d^{2}} \\
& i=1 m=1
\end{aligned}
$$

Donde:

$$
u=\frac{k_{x} d}{2}
$$

Simplificando (29), se tiene:

$$
\begin{gathered}
f_{y}{ }_{j}=4 \frac{\operatorname{sen}(u)}{k_{x}} \frac{\operatorname{sen}(v)}{k_{y}} \\
\sum_{l=1}^{N} \sum_{m=1}^{N} A_{l m} e^{j \beta(l, m)+j k_{x} x(l, m)+k_{y} y(l, m)}
\end{gathered}
$$

Evaluando el denominador de la ecuación

Expresando $G_{j}$ en términos de direcciones espaciales:

$G_{j}=\frac{64 \pi\left(\frac{\operatorname{sen}\left(\frac{k d \operatorname{sen}(\theta) \cos (\phi)}{2}\right)}{k \operatorname{sen}(\theta) \cos (\phi)} \frac{\left.\operatorname{sen}\left(\frac{k d \operatorname{sen}(\theta) \operatorname{sen}(\phi)}{2}\right)\right)^{2}}{k \operatorname{sen}(\theta) \operatorname{sen}(\phi)}\left(\sum_{l=1}^{N} \sum_{m=1}^{N} A_{l m} e^{j \beta(l, m)+j k \sin (\theta) \cos (\phi) x(l, m)+j k \sin (\theta) \sin (\phi) y(l, m)}\right)^{2}\right.}{d^{2} \sum_{l=1}^{N} \sum_{m=1}^{N}\left[A_{m}\right]^{2}}$ 


$$
\begin{gathered}
\int_{S} E_{y}^{2} d x d y=\sum_{l=1}^{N} \sum_{m=1}^{N}\left[\int_{-d / 2}^{d / 2} \int_{-d / 2}^{d / 2} A_{l m}{ }^{2} d x d y\right] \\
\int_{S} E_{y}^{2} d x d y=\sum_{l=1}^{N} \sum_{m=1}^{N}\left[A_{l m}{ }^{2} d^{2}\right]
\end{gathered}
$$

y sustituyendo (34) y (32) en (27) se tiene:

Donde

$$
k=\left(\frac{2 \pi}{\lambda}\right)
$$

Conocida la ganancia $G_{j}$ en cada dirección j, evaluada con la ecuación (36), se procede a compararla con la ganancia deseada, a través de una función de costo $\psi$ :

$$
\psi=\sum_{j=1}^{M}\left|G_{j}-G D_{j}\right|^{2}
$$

Donde:

$G D_{\mathrm{j}}=$ ganancia deseada en la dirección j.

$M=$ número de direcciones donde la ganancia será optimizada.

A continuación, se realiza un proceso de optimización de fase con el objetivo de reducir dicha función de costo dada por la ecuación (38). La fórmula recursiva para la optimización de la fase $\beta$ es (Cherrette, 1989):

$$
\beta_{l, m}(h+1)=\beta_{l, m}(h)-\mu \operatorname{Grad}(\beta)_{l, m}
$$

Donde h es el número de iteración y h+ 1 la siguiente iteración.

Un método para escoger el valor de $\mu$ que funciona bien en la práctica, es valuarlo como el inverso de la magnitud del gradiente en (39) y multiplicarlo por 0.9 cada vez que la función de costo se incrementa (Charrete, 1989). Para calcular el gradiente de la función costo se usa la siguiente deducción, omitida hasta ahora en la literatura disponible:

$$
\begin{gathered}
\frac{\partial \psi}{\partial \beta_{l, m}}=\frac{\partial}{\partial \beta_{l, m}}\left[\sum_{j=1}^{M}\left|G_{j}-G D_{j}\right|^{2}\right]= \\
\sum_{j=1}^{M} \frac{\partial}{\partial \beta_{l, m}}\left[G_{j}-\left.G D_{j}\right|^{2}\right]
\end{gathered}
$$

Desarrollando cualquiera de los sumandos de la sumatoria finita de la ecuación (40) se tiene:

$$
\begin{gathered}
\frac{\partial}{\partial \beta_{1, m}}\left[G_{j}-\left.G D_{j}\right|^{2}\right]= \\
2\left(G_{j}-G D_{j}\right) \frac{\partial}{\partial \beta_{l, m}}\left[\left(G_{j}-G D_{j}\right)\right]
\end{gathered}
$$

Como $\mathrm{GD}_{\mathrm{j}}$ es constante, la derivada del término derecho de la ecuación anterior, considerando la ecuación de ganancia (27), es:

$$
\begin{gathered}
\frac{\partial}{\partial \beta_{l, m}}\left[\left(G_{j}-G D_{j}\right)\right]=\frac{\partial}{\partial \beta_{l, m}} G_{j}= \\
\left.\frac{4 \pi}{d^{2} \sum_{l=1}^{N} \sum_{m=1}^{N}\left[A_{l m}\right]^{2}} \frac{\partial}{\partial \beta_{l, m}}\left|f_{y}\right|^{2}\right|_{j}
\end{gathered}
$$

La derivada del cuadrado de la transformada de Fou rier $f_{y}$ en (42) es:

$$
\begin{aligned}
& \left.\left.\frac{\partial}{\partial \beta_{l, m}} f_{y}\right|^{2}\right|_{j}=\frac{\partial}{\partial \beta_{l, m}}\left[f_{y} f_{y} *\right]= \\
& \mathrm{f}_{\mathrm{y}} \frac{\partial}{\partial \beta_{l, \mathrm{~m}}} \mathrm{f}_{\mathrm{y}} *+\mathrm{f}_{\mathrm{y}} * \frac{\partial}{\partial \beta_{l, \mathrm{~m}}} \mathrm{f}_{\mathrm{y}}
\end{aligned}
$$


Donde * in dica el complejo conjugado de la variable que le antecede. La derivada de la transformada de Fou rier $f_{y}$ es:

$\frac{\partial}{\partial \beta_{1, m}} f_{y}=A_{I m} d^{2} \frac{\operatorname{sen}(u)}{u} \frac{\operatorname{sen}(v)}{v} j e^{j \beta(l, m)+j k_{x} x(l, m)+j \mid j, y(l, m)}(44)$

Y la derivada del conjugado de dicha transformada de Fourier tiene la expresión siguiente:

$$
\frac{\partial}{\partial \beta_{l, m}} f_{y} *=
$$

$-A_{l m} d^{2} \frac{\operatorname{sen}(u)}{u} \frac{\operatorname{sen}(v)}{v} j e^{-j \beta(l, m)-j k_{x} x(l, m)-j k_{y} y(l, m)}$

(45)

Sustituyendo las ecuaciones (41) a la (45) en (40), se tiene la expresión final:

$$
\frac{\partial \psi}{\partial \beta_{l, m}}=\sum_{j=1}^{M} \frac{8 \pi}{d^{2} \sum_{l=1}^{N} \sum_{m=1}^{N}\left[A_{l m}\right]^{2}}\left(G_{j}-G D_{j}\right)
$$

$$
\left(A_{l m} d^{2} \frac{\operatorname{sen}(u)}{u} \frac{\operatorname{sen}(v)}{v}\right) X
$$

$\left\lfloor-j f_{y} e^{-j \beta l_{m}-j k_{x} x l_{m}-j k_{y} y_{m}}+j f_{y} * e^{j \beta l_{m}+j k_{x} x_{m}+j k_{y} y_{m}}\right\rfloor$

Esta ecuación expresa los elementos del gradiente de la función costo, respecto a la fase de cada elemento del plano de apertura, por lo que se puede aplicar la fórmula (39) de forma iterativa.

La optimización de fase puede consistir de muchas iteraciones, dependiendo de las condiciones del problema y de su comportamiento. En la gráfica de la figura 13

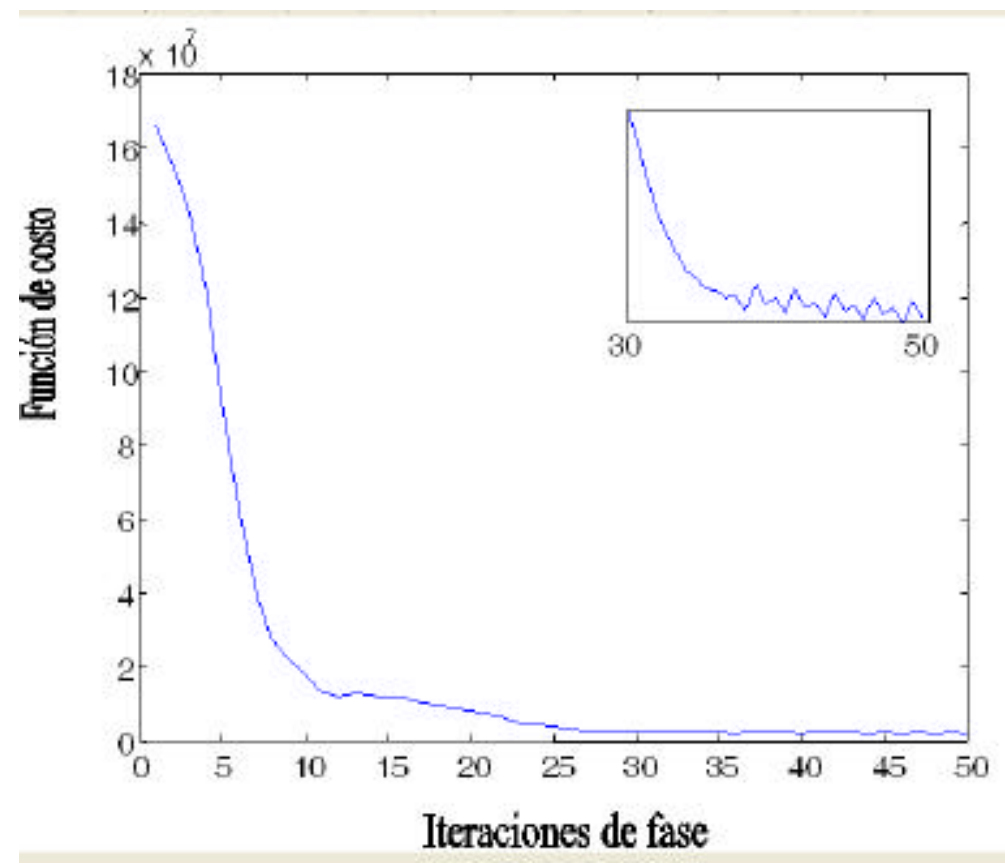

Figura 13. Comportamiento de la optimización de fase. Función costo vs número de iteraciones consecutivas de fase, sin hacer todavía ninguna corrección de amplitud del campo eléctrico en el plano de análisis. 
puede observarse el comportamiento típico de la función costo (y por ende, la cercanía a la optimización de fase) y cómo en las últimas iteraciones se presenta un fenómeno oscilatorio, pero cuasiestático; es decir, que no se obtiene ninguna mejora al aumentar el número de iteraciones después de cierto rango. Si para un cierto ejemplo o diseño se considera aceptable detenerse, digamos, después de la iteración 30 , se procede a calcular la nueva superficie del reflector parabólico perfilado, como se in dica a continuación.

\section{Análisis matemático ( $3 \stackrel{a}{a}$ parte)}

El número de iteraciones para optimizar la fase minimizando la función de costo, depende directamente del número de elementos en la rejilla, del número de direcciones de optimización y del rango entre el promedio de la ganancia inicial y la ganancia deseada en las direcciones de optimización. Para una cierta huella pueden ser suficientes $\begin{array}{llll}30 & 0 & 40\end{array}$ iteraciones, pero para otras puede llegarse

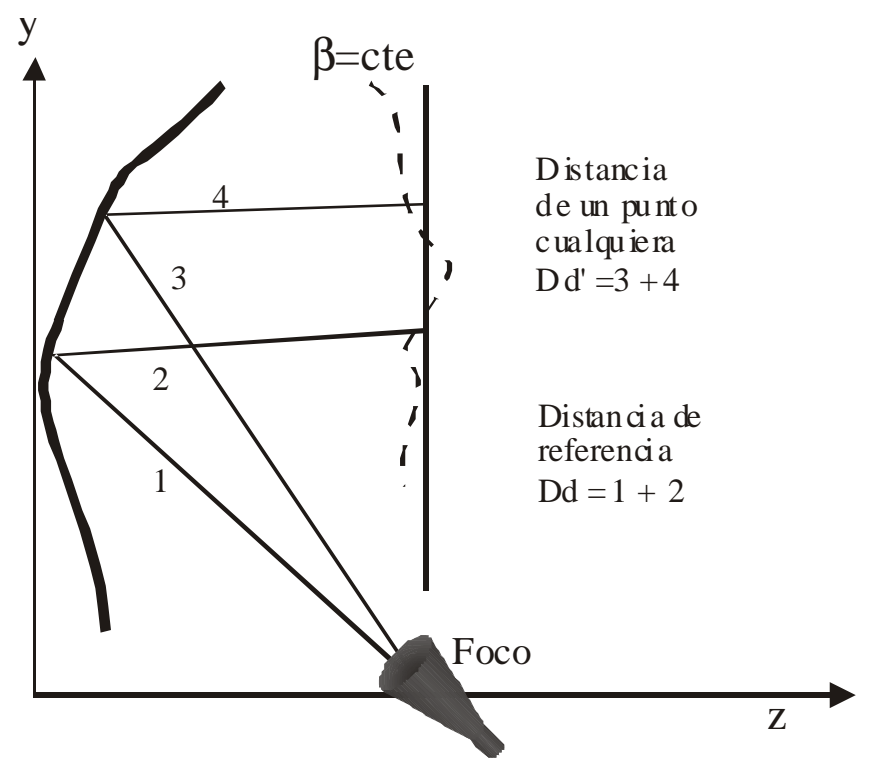

hasta 100 o más. Con esta nueva distribución de fase se procede entonces a modificar la superficie del re flec tor.

La nueva superficie del reflector debe calcularse a partir de la distribución final de fase, que debe equivaler a diferencias de fracciones de longitud de onda en la superficie del reflector. Tomando como referencia el punto focal y la distancia que recorre un rayo desde el foco hasta el plano de análisis, en una línea paralela al eje $z$, se tiene que:

$$
\begin{aligned}
& D d=2 f=\text { distancia de referencia. } \\
& \beta_{\text {ref }}=0=\text { fase de referencia. }
\end{aligned}
$$

$D d$ corresponde a la distancia entre el alimentador y el plano de análisis en dirección del eje z (Figura 14). Para cualquier otro punto, como se puede ver en la misma figura:

$$
\begin{gathered}
D d^{\prime}=\left[x_{s}^{2}+y_{s}^{2}+\left(z_{s}-f\right)^{2}\right]^{\frac{1}{2}}+ \\
{\left[\left(x_{s}-x\right)^{2}+\left(y_{s}-y\right)^{2}+\left(z_{s}\right)^{2}\right]^{\frac{1}{2}}}
\end{gathered}
$$

Figura 14. Definición de las distancias de referencia para el cálculo de la nueva superficie del reflector parabólico perfilado. La línea curva punteada sobre el plano de análisis es una curva de fase constante 
Donde $\left(x_{s}, y_{s}, z_{s}\right)$ es un punto del re flec tory $(x$, $y, 0)$ es un punto del plano de análisis.

Para que el reflector produzca las diferencias de fase, la siguiente relación debe mantenerse (Figura 14):

$$
D d^{\prime}=\frac{-1}{k}\left[\beta-\beta_{r e f}\right]+D d
$$

Con la finalidad de calcular la dirección de cada rayo que incide sobre el plano de análisis, se demuestra a continuación que los rayos reflejados son normales a las curvas de equifase de la distribución, lo cual permite conocer las pendientes $m$ de cada rayo. Las componentes de la pendiente de cada rayo están dadas por las componentes del vector de propagación $k$ normalizado, es decir:

$$
\begin{gathered}
m_{x}=\frac{k_{x}}{k}{ }_{(x, y, z)} \\
m_{y}=\frac{k_{y}}{k}{ }_{(x, y, z)} \\
m_{z}=\frac{k z}{k}_{(x, y, z)}
\end{gathered}
$$

Las curvas de equifase están caracterizadas por la siguiente ecuación de onda plana:

$\mathbf{k} \cdot \mathbf{r}-\beta=k_{x} x+k_{y} y+k_{z} z-\beta=$

Constante (52)

Y al obtener el gradiente de la ecuación (52) resultan las siguientes ecuaciones que comprueban lo dicho en el párrafo anterior:

$$
m_{x}=\left.\frac{k_{x}}{k}\right|_{(x, y, z)}=\left.\frac{1}{k} \frac{\partial \beta}{\partial x}\right|_{(x, y, z)}
$$

$$
\begin{gathered}
m_{y}=\left.\frac{k_{y}}{k}\right|_{(x, y, z)}=\frac{1}{k} \frac{\partial \beta}{\partial y} \\
m_{z}=\sqrt{1-m_{x}^{2}-m_{y}^{2}}
\end{gathered}
$$

Con las componentes anteriores se pueden relacionar las coordenadas de cada punto $(x, y$, $z$ ) en el plano de análisis con su punto correspondiente $\left(x_{s}, y_{s}, z_{s}\right)$ en la superficie del reflector:

$$
\begin{aligned}
& \frac{x_{s}-x}{m_{x}}=\frac{z_{s}-z}{m_{z}} \\
& \frac{y_{s}-y}{m_{y}}=\frac{z_{s}-z}{m_{z}}
\end{aligned}
$$

La solución de las ecuaciones (47), (56) y (57) está dada finalmente por:

$$
\begin{gathered}
Z_{S}=\frac{Q^{2}(x, y)-x^{2}-y^{2}-f^{2}}{2(\sigma x+\gamma y-f)-2 Q(x, y)\left(\sigma^{2}+\gamma^{2}+1\right)^{1 / 2}} \\
y_{S}=\gamma\left(z_{S}\right)+y
\end{gathered}
$$

$$
x_{s}=\sigma\left(z_{s}\right)+x
$$

Donde

$$
\begin{gathered}
Q(x, y)=\frac{1}{k}\left[-\beta_{l, m}\right]+D d \\
\gamma=\frac{m_{y}}{m_{z}}
\end{gathered}
$$

$$
\sigma=\frac{m_{x}}{m_{z}}
$$


Los valores de $z_{s}$ calculados con la ecuación (58) pueden verse afectados por variaciones bruscas de la fase, por lo que pueden aplicarse tolerancias y límites para que los valores de la nueva superficie del reflector no salgan más allá de cierta región en el espacio (Shoki, 1993).

Una vez calculada la superficie perfilada del reflector, se puede proceder a obtener la nueva distribución de amplitud del campo eléctrico en el plano de análisis. El método descrito por Cherrette (1989) utiliza la relación entre las áreas del plano y del reflector para calcular la amplitud en cada punto del plano:

$$
\left|E_{a}\right|=\left|E_{r}\right| \sqrt{\frac{\text { área }}{\text { área }}}
$$

Donde

$\left.E_{r}\right|_{=}$magnitud del campo reflejado en la superficie del reflector.

$\mathbb{E}_{\mathrm{a}}=$ magnitud del campo reflejado en el plano de análisis.

área $=$ área proyectada por un rayo en forma de tubo en la superficie del reflector.

área $=$ área proyectada por un rayo en forma de tubo en el plano de análisis.

Suponiendo que el área proyectada en el re flec tor es un triángulo:

$$
\text { área }_{1}=\frac{1}{2} h(\text { lado } 1) \mathbf{k}_{\mathbf{i}} \cdot \mathbf{n}
$$

Donde:

$$
h=(l a d o 2) \sqrt{1-(\cos \alpha)^{2}}
$$

$$
\cos \alpha=\frac{(\text { lado3 })^{2}-(\text { lado } 1)^{2}-(\text { lado2 })^{2}}{2(\text { lado })(\text { lado } 2)}
$$

Lado $I=|P(I, J)-P(I+1, J)|$

Lado $2=|P(I, J)-P(I, J+1)|$

Lado $3=|P(I+1, J)-P(I, J+1)|$

$\mathbf{K}_{\mathbf{i}}=$ dirección del rayo incidente

$\mathbf{n}=$ normal a la superficie en el punto $P(I, J)$.

$$
\text { área }_{2}=\frac{1}{2} \text { (célula) } \mathbf{k}_{\mathbf{r}} \cdot \mathbf{z}
$$

célula = área de un elemento en el plano de análisis.

$\mathbf{K}_{\mathbf{r}}=$ dirección normalizada del rayo reflejado.

Con las nuevas distribuciones de fase y amplitud en el plano de análisis, es posible calcular una nueva distribución de ganancias del campo radiado. La ganancia en cada dirección hacia la zona geográfica de servicio se compara con la ganancia deseada. Si las diferencias entre ambas ganancias no son satisfactorias se repite todo el proceso iterativo cuantas veces sea necesario, hasta que el patrón se conforme lo más posible a las características de la huella especificada.

\section{Ejemplo de una antena perfilada para el territorio nacional mexicano}

Se aplicará ahora el método descrito para el análisis de una huella conformada para el territorio mexicano. El análisis se realiza con dimensiones en longitudes de onda y puede aplicarse a cualquier banda de frecuencias, a excepción de que exista un límite físico en 
cuanto al tamaño del plato. Siguiendo los pasos mencionados al inicio de este artículo:

1) México, como se observa en la figura 15 , cuenta con una geografía complicada y con muchas irregularidades. Es conveniente no tratar con irregularidades tales como la península de Baja California, o con cavidades como el Golfo de México, por lo que se define un contorno suave como el mostrado en la figura. El territorio mexicano alcanza un máximo de cerca de 5 grados de ancho y un mínimo de aproximadamente 3 grados. Desde luego, se tomó en cuenta el centro del área de servicio con su latitud geográfica correcta, tal como se aclaró en el procedimiento gen eral al inicio de este artículo. Considerando un mínimo ancho del haz de 3 grados y tomando en cuenta la ecuación (1), el diámetro del reflector necesario es de $25 \lambda$. Los demás datos utilizados son: Distancia focal $f=25 \lambda$, expo- nente del alimentador $q=11$ y ganancia deseada en los puntos de optimización: 31dB (con estos parámetros se obtiene el círculo grueso de la huella en la figura 17). Las direcciones de optimización se definieron sobre un contorno suave (38 direcciones) y en un número suficiente de puntos para definir el contorno de ganancia deseado.

2) Una vez definido el reflector inicial se calcula su ganancia (Figura 16) y se puede observar su huella de radiación simétrica (Figura 17). A continuación se procede a minimizar la función de costo y obtener la distribución de fase óptima (Figura 18). Se hicieron 60 iteraciones consecutivas.

3) A partir de la nueva distribución de fase se calcula una nueva superficie del reflector y en la figura 19 se muestran las curvas de nivel de la diferencia con el re flec tor orig i nal.

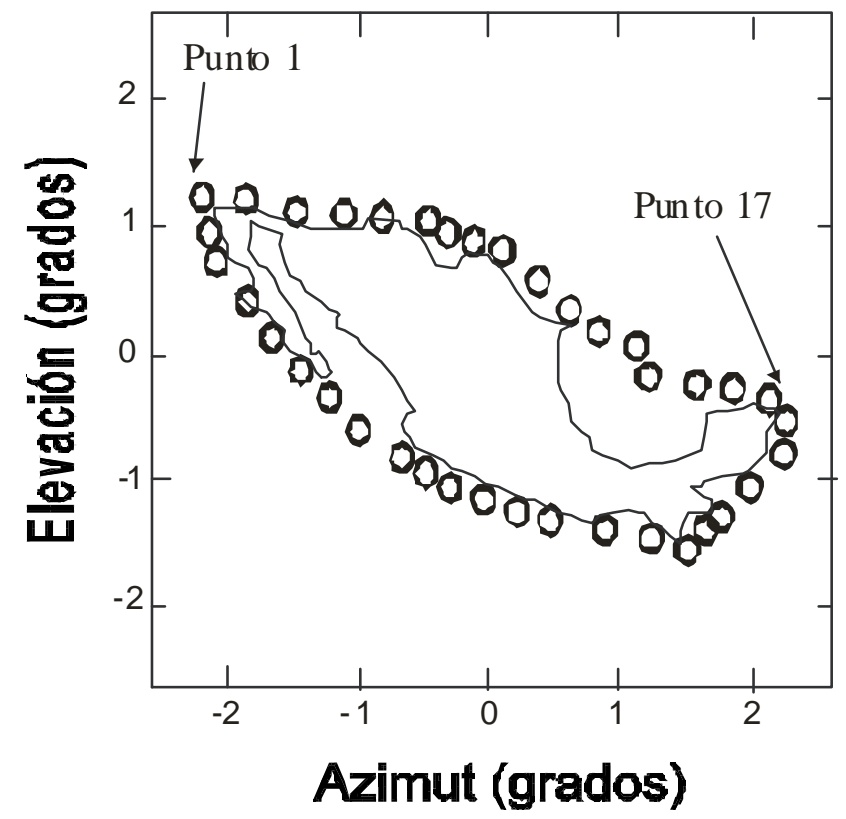

Figura 15. Definición de la zona de cobertura y de las direcciones de optimización. La numeración de los círculos avanza en el sentido de las manecillas del reloj. Estos círculos delimitan el contorno deseado de - $3 \mathrm{~dB}$ con relación a la ganancia máxima en el centro del país. Los grados de elevación y azimut están medidos con relación al eje prin cipal de la antena parabólica perfecta inicial; es decir, el eje z apuntando hacia el punto $(0,0)$. Las correcciones de latitud ya están consideradas 


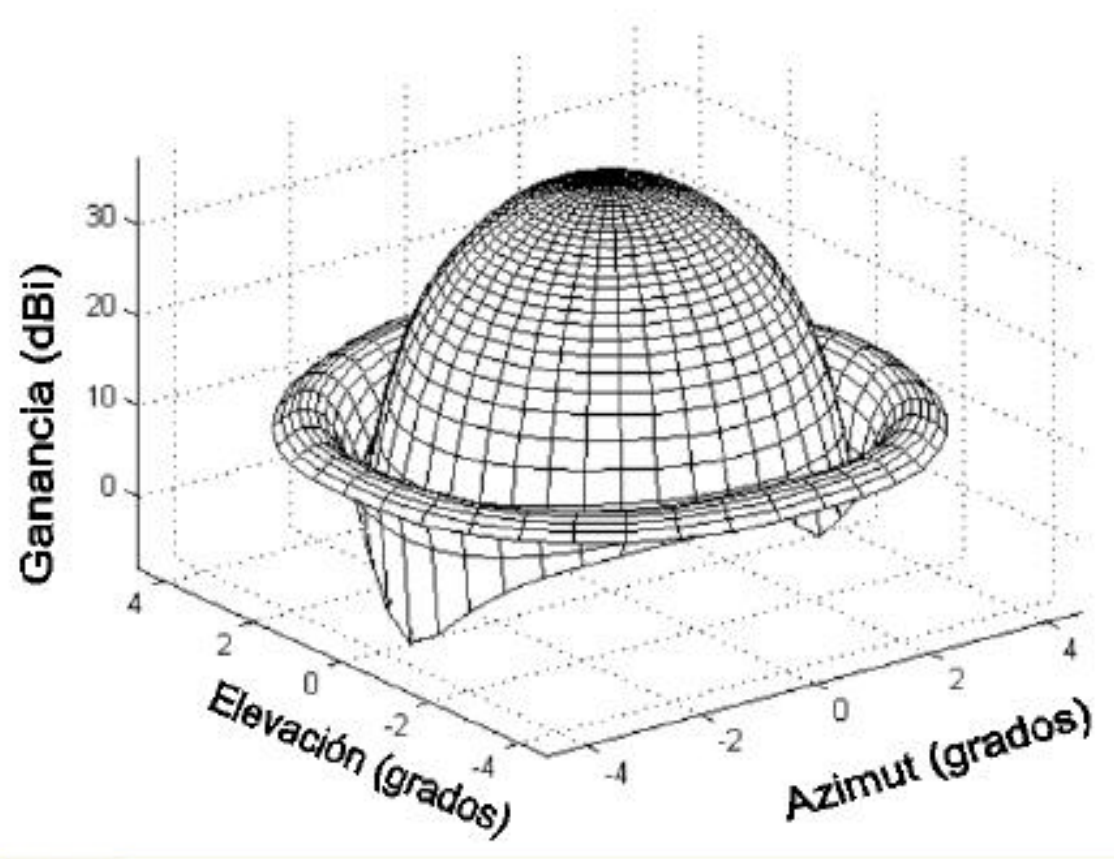

Figura 16. Patrón tridimensional del reflector inicial. $G$ anancia $=10 \log \left(G_{j}\right)$

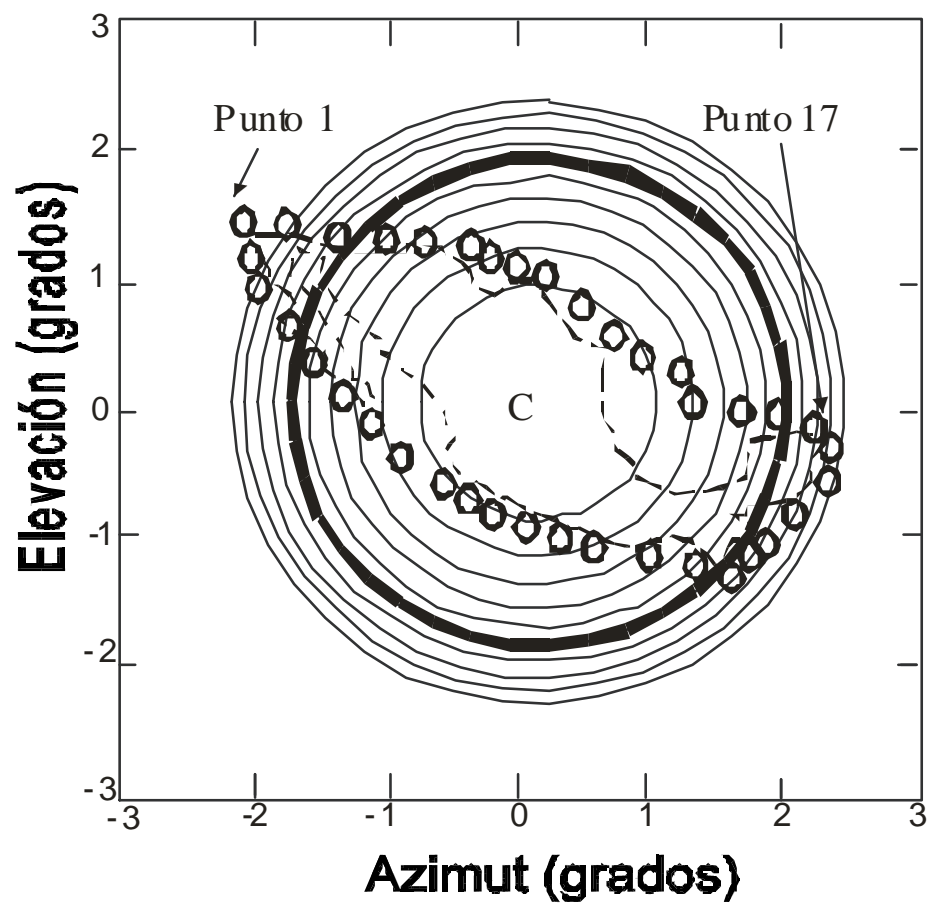

Figura 17. Huella simétrica de radiación producida por el reflector inicial. El círculo grueso corresponde a los puntos hacia donde la ganancia radiada es igual a $31 \mathrm{dBi}$. 


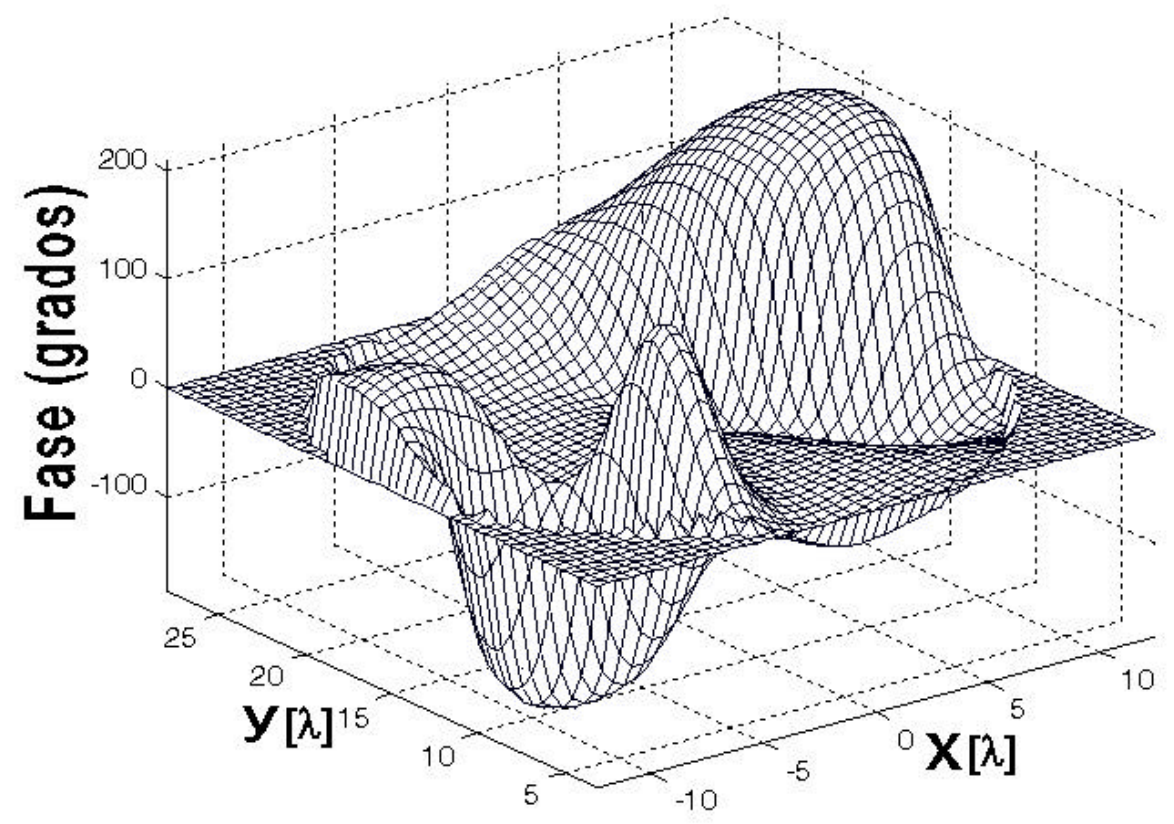

Figura 18. Distribución de fase después de 60 iteraciones consecutivas de fase

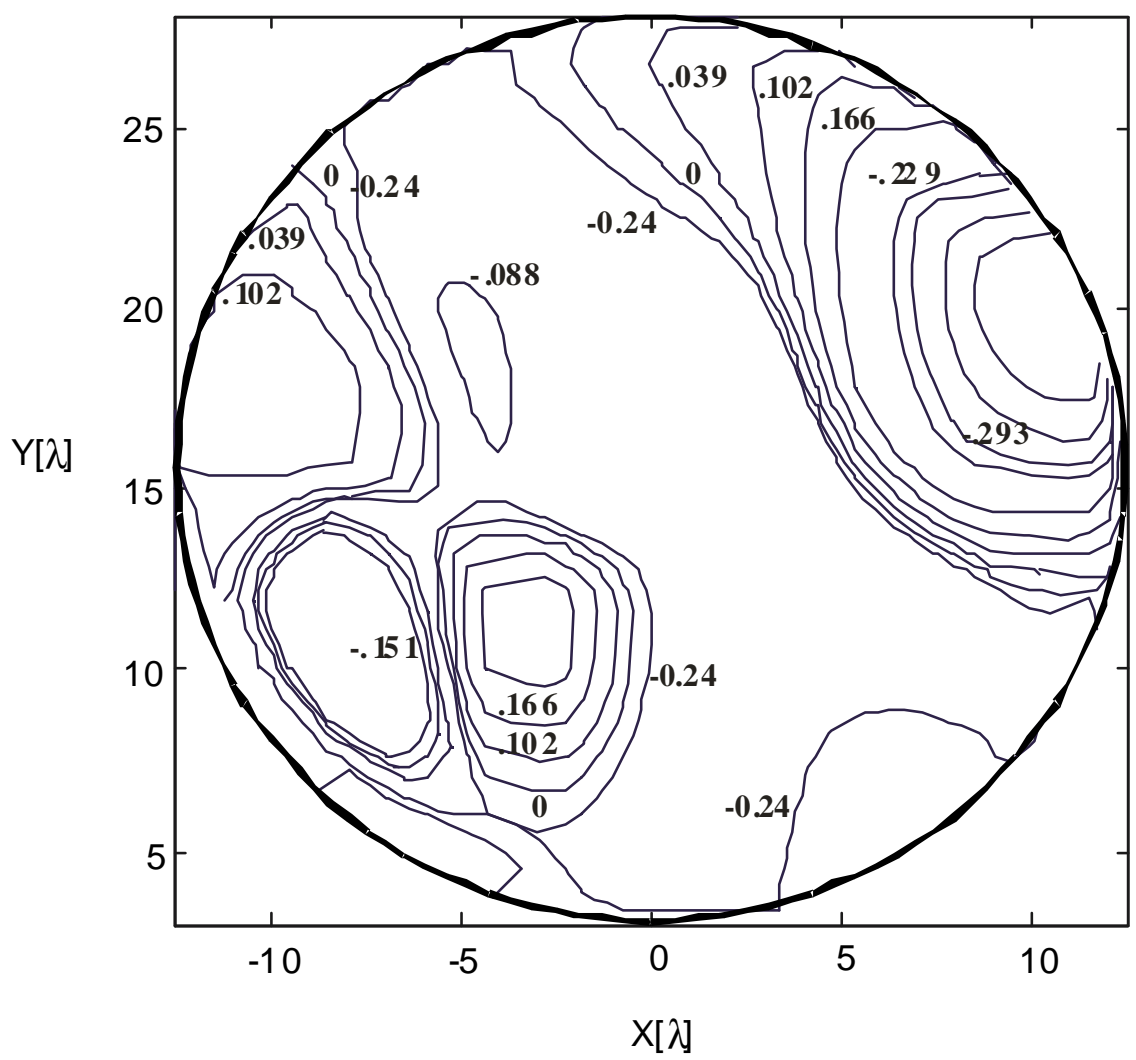

Figura 19. Curvas de nivel de la diferencia (en $\lambda$ ) entre la nueva superfice del reflector y el reflector orig inal en la dirección z. Los resultados fueron obtenidos a partir de una rejilla de $50 \times 50$ elementos y 60 iteraciones consecutivas de fase 


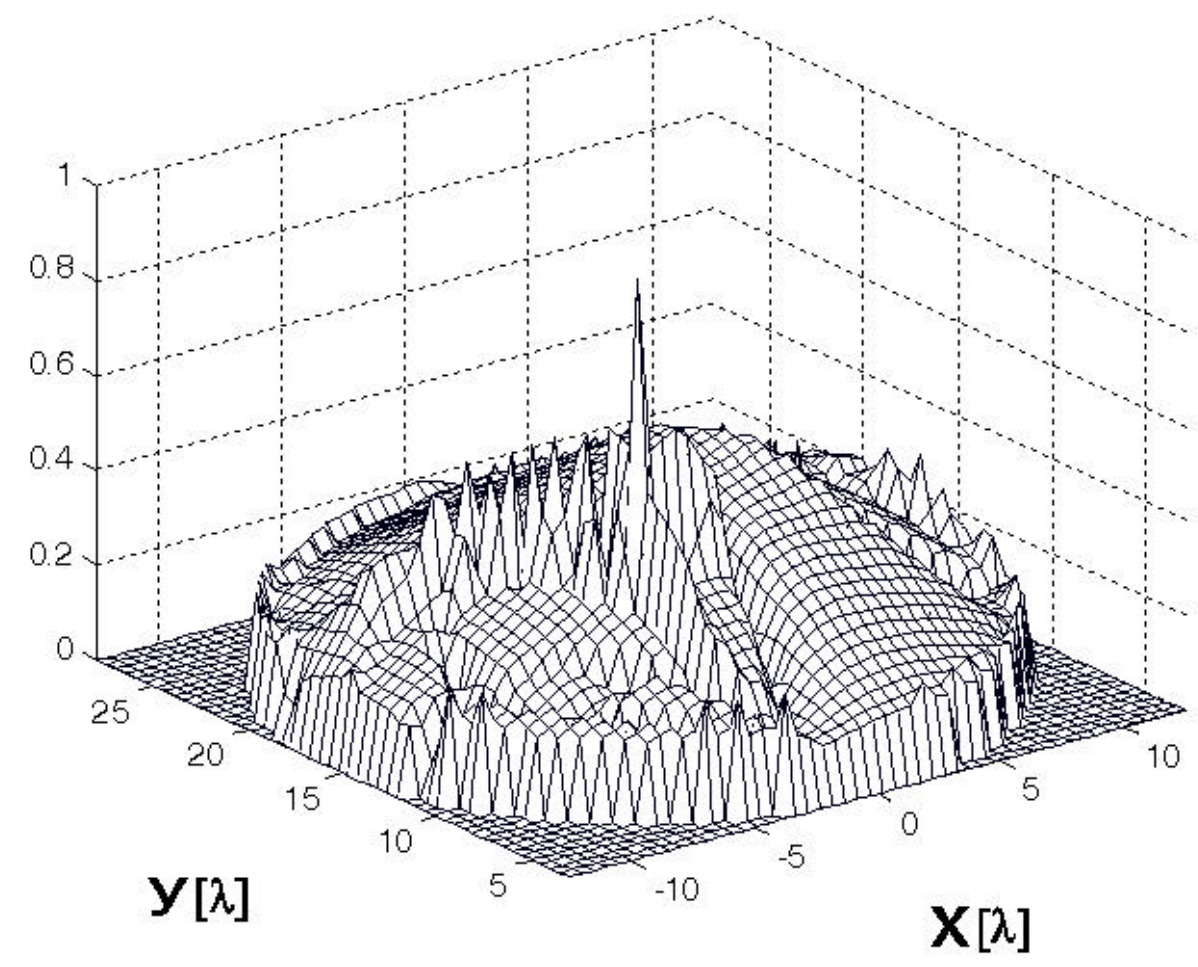

Figura 20. Distribución de amplitud corregida en el plano de análisis a partir de la superficie del reflector de la figura 19. El eje vertical indica la amplitud normalizada de la intensidad de campo eléctrico

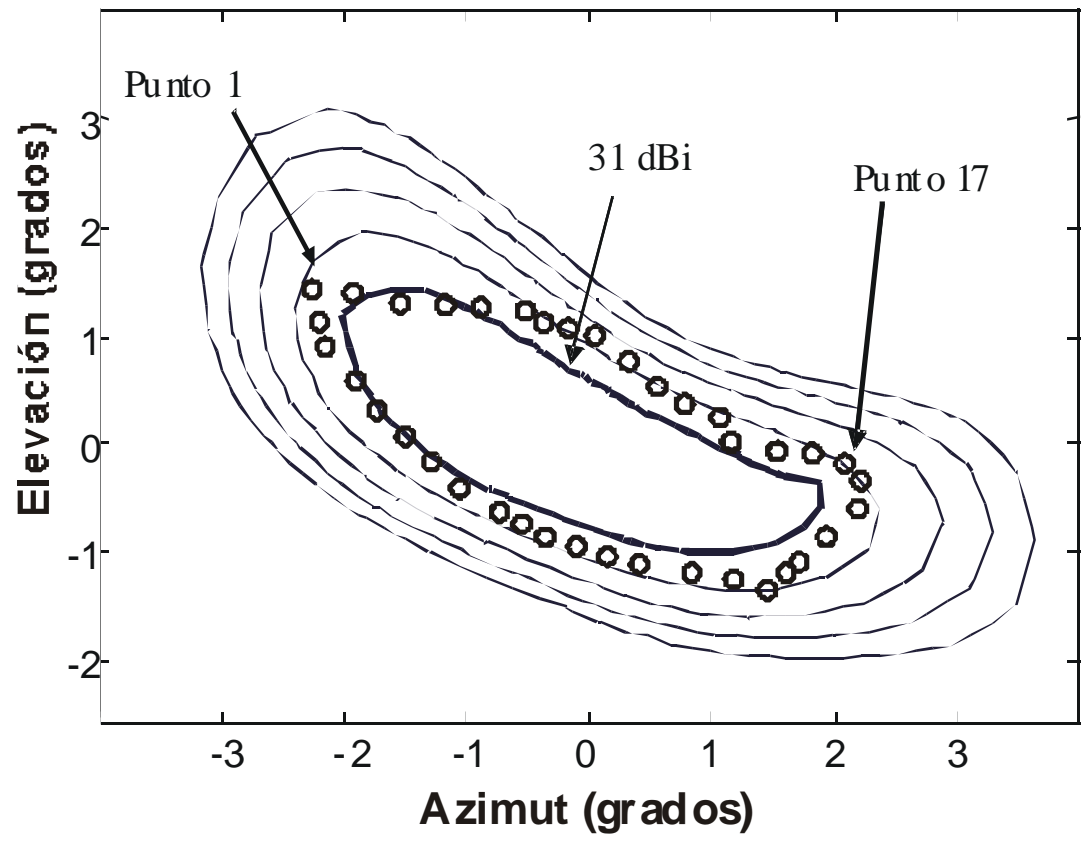

Figura 21. Huella de radiación del reflector parabólico perfilado después de 60 iteraciones consecu tivas de fase y una corrección de amplitud. La diferencia de ganancia entre contornos adyacentes es $1 \mathrm{~dB}$, disminuyendo hacia fuera del contorno grueso de $31 \mathrm{dBi}$ 


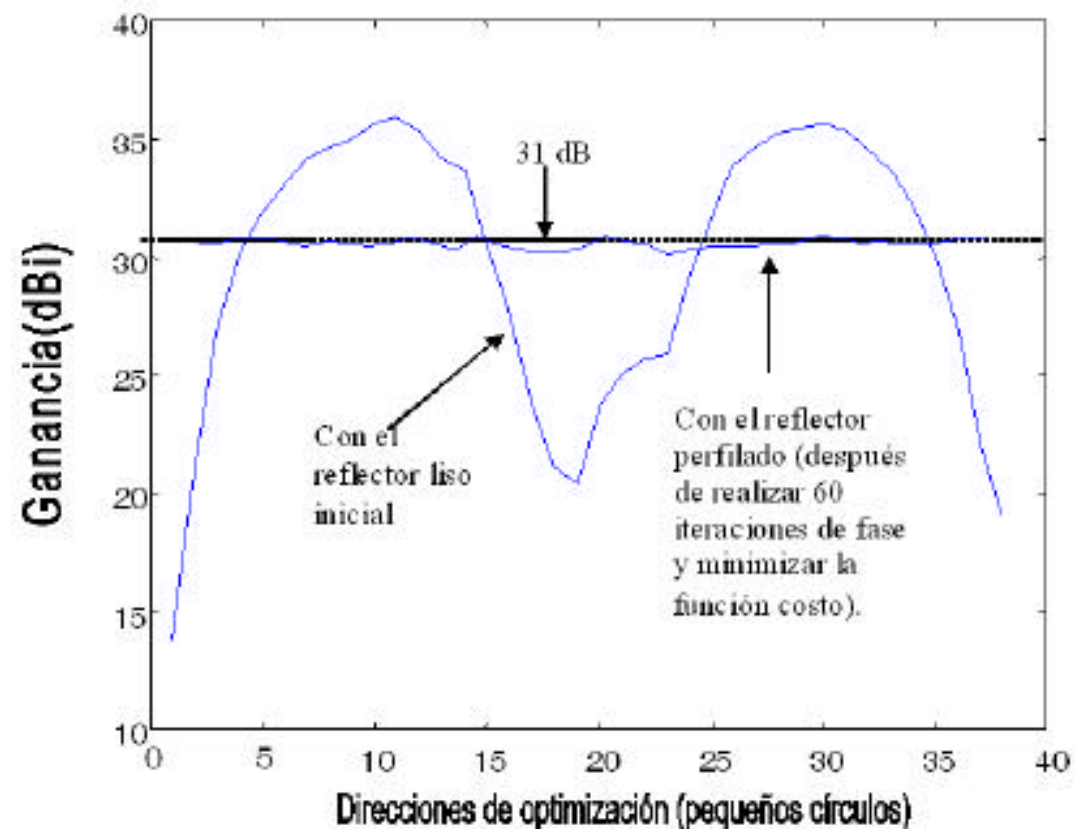

Figura 22. Función de ganancia hacia las direcciones de optimización antes y después de modificar el reflector amplitud del campo eléctrico en el plano de análisis (Figura 20).

5) Se calcula la nueva ganancia en varios puntos dentro y fuera del contorno establecido, originalmente para obtener la huella de radiación. Ésta se muestra en la figura 21. Como se observa, se obtuvo una huella bastante aproximada a la zona de cobertura. Por último, en la figura 22 se muestran comparativamente las ganancias obtenidas en el contorno especificado antes y después de perfilar el plato.

\section{Conclusiones}

El método presentado ha sido aplicado con éxito al ejemplo de México y se han desarrollado las fórmulas matemáticas necesarias para programarlo con facilidad y obtener resultados con diferentes parámetros. Se incluyeron gráficas y figuras auxiliares, así como guías sobre el comportamiento del método iterativo, que no se proporcionan o especifican con claridad en otras publicaciones disponibles; por ejemplo, el número de iteraciones necesarias y la conveniencia de definir un nivel adecuado de ganancia como condición inicial para que el método converja de una forma efectiva. El presente trabajo sirve como referencia técnica y matemática tanto para la docencia como para futuras investigaciones.

\section{Referencias}

Clarricoats P.J. (1984). Corrugated Horns for Microwave Antennas. Ed. Peter Peregrinus, Reino Unido.

Collin R.E. (1985). Antennas and Radiowave Propagation. McGraw-Hill.

Cherrette A.R., Lee S. y Acosta R.J. (1989). A Method for Producing a Shaped Contour Radiation Pattern Using a Single Shaped Reflector and a Single Feed. IEEE Trans. On Antennas and Prop agation, Vol. 37, No. 6, pp 698-706. 
Neri R. (2003). Comunicaciones por satélite. Ed. Thomson Learning, México.

Pinsard B., Renaud D. y Diez H. (1997). New Surface Expan sion for Fast PO Synthesis of Shaped Reflector Antennas. 10th International Conference on Antennas and Propagation, Abril 14-17. Publicación No. 436 del IEEE, Vol. 1, pp 25-29. Sámano R., Mendoza I. y Neri R. (2002). Programación óptima para obtener patrones tridimensionales de antenas de apertura con la Transformada Discreta de Fourier. Revista Ingeniería Investigación y Tecnología, UNAM, Vol. III, No.3, Julio-Septiembre 2002, pp 93-100.
Shogen K., Nishida H. y Toyama N. (1992). Single Shaped Reflector Antennas for Broadcasting Satellites. Trans. on Antennas and Propagation, Vol. 40, No. 2, pp 178-187.

Shoki H. y Kawabata K. (1993). A Multiple Shaped Beam Antenna Using a Single Shaped Reflector. IEICE Trans. on Communications, Vol. E76-B, No. 12, Japón, pp. 1500-1507.

Volakis J.L. (1998). SABOR: Description of the Methods Applied for a Fast Anal ysis of Hornand Reflector Antennas. IEEE Antennas and Propagation Magazine, Vol. 40, No. 4.

\section{Semblanza de los autores}

Salvador Landeros-Ayala Realizó estudios de ingeniería eléctrica en la Universidad Nacional Autónoma de México en 1974. En 1976 obtuvo su maestría en comunicaciones por la Universidad de Pennsylvania, USA y el doctorado por la UNAM en 1999. Ha sido profesor durante más de 20 años en la Facultad de Ingeniería en materias como antenas, enlaces de microondas, propagación de radio y sistemas satelitales.

Ramiro Sámano-Robles. Obtuvo su título como ingeniero en telecomunicaciones con mención honorífica en la Facultad de Ingeniería, UNAM. Perteneció al Programa de Alto Rendimiento Académico de la misma Facultad. En el 2003, logró la maestría en telecomunicaciones en la Universidad de Essex, Inglaterra becado por el Consejo Nacional de Ciencia y Tecnología. Se ha desempeñado profesionalmente en el área de comunicaciones móviles (UNEFON) y en el diseño de circuitos de RF (Kb/TEL Telecomunicaciones).

Rodolfo N eri-Vela Realizó los estudios de ingeniero mecánico electricista y se especializó en telecomunicaciones y electrónica en la Facultad de Ingeniería, UNAM. En 1976, terminó la maestría en telecomunicaciones en la Universidad de Essex, Inglaterra, becado por el Consejo Británico. Tres años después obtuvo el grado de doctor en radiación electromagnética aplicada por la Universidad de Birmingham, Inglaterra, como becario del Consejo Nacional de Ciencia y Tecnolo gía (CONACYT). En 1985, se convirtió en el primer astronauta mexicano al participar en la misión 61-B de la NASA de los EU y orbitar la Tierra 109 veces. Actualmente imparte cátedra en el Departamento de Telecomunicaciones de la Facultad de Ingeniería de la UNAM. 